\title{
SoxC Transcription Factors Are Required for Neuronal Differentiation in Adult Hippocampal Neurogenesis
}

\author{
Lifang $\mathrm{Mu},{ }^{1 \star}$ Lucia Berti, ${ }^{1 \star}$ Giacomo Masserdotti, ${ }^{3,6 \star}$ Marcela Covic, ${ }^{1}$ Theologos M. Michaelidis,,${ }^{1,4}$ Kathrin Doberauer, ${ }^{1}$ \\ Katharina Merz, ${ }^{1}$ Frederick Rehfeld, ${ }^{1}$ Anja Haslinger, ${ }^{1}$ Michael Wegner, ${ }^{2}$ Elisabeth Sock, ${ }^{2}$ Veronique Lefebvre, ${ }^{5}$ \\ Sebastien Couillard-Despres, ${ }^{7}$ Ludwig Aigner, ${ }^{7}$ Benedikt Berninger, ${ }^{3,6}$ and D. Chichung Lie $^{1,2}$ \\ ${ }^{1}$ Research Group Adult Neurogenesis and Neural Stem Cells, Institute of Developmental Genetics, Helmholtz Zentrum München, German Research Center \\ for Environmental Health, Munich-Neuherberg, Germany, ${ }^{2}$ Institute of Biochemistry, Emil Fischer Center, University Erlangen-Nürnberg, Erlangen, \\ Germany, ${ }^{3}$ Department of Physiological Genomics, Institute of Physiology, Ludwig-Maximilians University Munich, Munich, Germany, ${ }^{4}$ Foundation for \\ Research and Technology-Hellas, Biomedical Research Institute, University of Ioannina, Science and Technology School, 45110 Ioannina, Greece, ${ }^{5}$ Lerner \\ Institute, Cleveland Clinic, Cleveland, Ohio, ${ }^{6}$ Institute for Stem Cell Research, National Research Center for Environment and Health, Munich-Neuherberg, \\ Germany, and 7Institute of Molecular Regenerative Medicine, Paracelsus Medical University Salzburg, Austria
}

Neural stem cells (NSCs) generate new hippocampal dentate granule neurons throughout adulthood. The genetic programs controlling neuronal differentiation of adult NSCs are only poorly understood. Here we show that, in the adult mouse hippocampus, expression of the SoxC transcription factors Sox 4 and Sox 11 is initiated around the time of neuronal commitment of adult NSCs and is maintained in immature neurons. Overexpression of Sox 4 and Sox 11 strongly promotes in vitro neurogenesis from adult NSCs, whereas ablation of Sox4/Sox 11 prevents in vitro and in vivo neurogenesis from adult NSCs. Moreover, we demonstrate that SoxC transcription factors target the promoters of genes that are induced on neuronal differentiation of adult NSCs. Finally, we show that reprogramming of astroglia into neurons is dependent on the presence of SoxC factors. These data identify SoxC proteins as essential contributors to the genetic network controlling neuronal differentiation in adult neurogenesis and neuronal reprogramming of somatic cells.

\section{Introduction}

The hippocampal dentate gyrus is one of two regions in the mammalian brain, in which neural stem cells (NSCs) give rise to new neurons throughout adulthood. NSCs produce dentate granule neurons through a stereotypic sequence of proliferation and differentiation steps. Cells of different developmental stage in the adult neurogenic lineage express distinct sets of marker proteins and display striking morphological and electrophysiological differences (Zhao et al., 2008), indicating that

Received Sept. 12, 2011; revised Dec. 22, 2011; accepted Jan. 11, 2012.

Author contributions: L.M., L.B., G.M., M.C., T.M.M., K.D., K.M., F.R., A.H., B.B., and D.C.L. designed research; L.M., L.B., G.M., M.C., T.M.M., K.D., K.M., F.R., and A.H. performed research; M.W., E.S., V.L., S.C.-D., and L.A. contributed unpublished reagents/analytic tools; L.M., L.B., G.M., M.C., T.M.M., K.D., K.M., F.R., A.H., B.B., and D.C.L. analyzed data; D.C.L. wrote the paper.

K.D. is supported by a graduate student fellowship of the Technical University Munich. Work in the Lie laboratory is supported by the European Young Investigator Award of the European Science Foundation (DFG 858/6-2), the Marie Curie Excellence Team Program and the Marie Curie Reintegration Program of the European Union, the Bavarian Research Network on Adult Neural Stem Cells "FORNEUROCELL," the Helmholtz Alliance for Mental Health in an Ageing Society, the Bundesministerium für Bildung und Forschung (BMBF) Network "Cell-Based Regenerative Medicine," and the European Commission Coordination Action ENINET (Network of European Neuroscience Institutes) Contract LSHM-CT-2005-19063). The work of G.M. and B.B. was supported by Deutsche Forschungsgemeinschaft Grant BE 4182/2-2 and the BMBF network "Neurogenesis from Brain and Skin Cells-New Approaches towards Neuronal Regeneration" (B.B.). We thank K. Wassmer, M. Ram, and F. Gruhn for excellent technical support and members of the Lie laboratory for helpful discussion and suggestions. We are grateful to Dr. S. Jessberger for discussion and comments on this manuscript. We also thank Dr. H. Kondoh for providing cDNAs for Sox4 and Sox 11.

*L.M., L.B., and G.M. contributed equally to this work.

Correspondence should be addressed to D. Chichung Lie, Institute of Biochemistry, Emil Fischer Center, University Erlangen-Nürnberg, 91051 Erlangen, Germany. E-mail: c.lie@biochem.uni-erlangen.de.

DOI:10.1523/JNEUROSCI.4679-11.2012

Copyright $\odot 2012$ the authors $\quad 0270-6474 / 12 / 323067-14 \$ 15.00 / 0$ stage-specific genetic programs underlie the development of new hippocampal neurons.

A crucial step in adult hippocampal neurogenesis is the commitment of precursor cells to a neuronal fate. Neuronal fate commitment is accompanied by downregulation of the multipotency factor Sox2 (Ellis et al., 2004; Steiner et al., 2006) and expression of the transcription factors NeuroD1 (Steiner et al., 2006; Gao et al., 2009) and Prox1 (Steiner et al., 2008; Lavado et al., 2010). These transcription factors are regulated by the Wnt-signaling pathway (Kuwabara et al., 2009; Karalay et al., 2011) whose activity is required for the generation of new hippocampal neurons during adulthood (Lie et al., 2005). Loss of NeuroD1 (Gao et al., 2009) and Prox1 (Lavado et al., 2010; Karalay et al., 2011) in the hippocampal neurogenic lineage results in impaired maturation and survival of new neurons. Notably, NeuroD1-deficient (Gao et al., 2009) and Prox1-deficient (Lavado et al., 2010) NSCs do give rise to immature neurons, indicating that additional genetic programs contribute to neuronal fate commitment of NSCs in the adult hippocampus.

The high-mobility group box proteins Sox 4 , Sox 11 , and Sox 12 constitute the SoxC family of transcription factors. SoxC factors are potent regulators of mammalian nervous system development and have been found to control survival of neural precursors and their differentiated progeny in a highly redundant manner (Hoser et al., 2008; Bhattaram et al., 2010; Potzner et al., 2010; Thein et al., 2010). In addition, a recent genome-wide binding study demonstrated enrichment of Sox11 on neuron-specific genes in embryonic stem cell-derived neurons (Bergsland et al., 
2011), indicating that SoxC factors may be key regulators for neuronal differentiation of mammalian NSCs.

We previously found that Sox 11 is almost exclusively expressed in neurogenic niches of the adult mammalian brain. Intriguingly, the initiation of Sox11 expression in the adult neurogenic lineage coincides with the loss of Sox 2 and the onset of the expression of neuron-specific proteins (Haslinger et al., 2009). Here, we investigate the hypothesis that SoxC proteins are essential regulators of neuronal fate commitment in adult hippocampal neurogenesis. We demonstrate that Sox4 and Sox11 exhibit highly overlapping expression in the hippocampal neurogenic lineage and that overexpression of either SoxC factor is sufficient to induce neuronal marker expression in adult NSCs. Conversely, loss of Sox4/Sox11 results in loss of expression of neuron-specific proteins in vitro and in vivo. Promoter studies reveal that SoxC factors directly control the activity of neuronspecific gene promoters in adult NSCs. Finally, we demonstrate that SoxC proteins do not only fulfill essential functions in neuronal differentiation in adult hippocampal neurogenesis but that they are also vital for efficient neuronal reprogramming of somatic cells.

\section{Materials and Methods}

Animals. All experiments were performed in accordance with the European Communities Council Directive (86/609/EEC). Stereotactic injections of retroviruses into the brain of adult mice were approved by the Government of Upper Bavaria. For all experiments, 8- to 12-week-old mice were used. Mice were grouped housed in standard cages under a $12 \mathrm{~h}$ light/dark cycle and had ad libitum access to food and water. C57BL/6 mice from Charles River were used for expression analysis of SoxC transcription factors. Loss-of-function experiments were performed on mice of mixed 129SvJ/C57Bl6J background carrying the following Sox 4 and Sox 11 alleles: Sox $4^{\text {loxP }}$ (Penzo-Méndez et al., 2007) and Sox11 $1^{\text {loxP }}$ (Bhattaram et al., 2010).

For loss of Sox4/Sox11 function experiments, at least six animals per group were analyzed. Male and female littermates were included in the analysis. Experimental groups were matched for age and sex.

Retrovirus preparation. The $\mathrm{pCAG-GFP}$ and $\mathrm{pCAG}-\mathrm{RFP}$ retroviruses have been described previously (Zhao et al., 2006; Jagasia et al., 2009). For retrovirus-mediated expression of Sox 4 and Sox11, their respective cDNAs were cloned into the pCAG-IRES-GFP (Jagasia et al., 2009) to generate pCAG-Sox4-IRES-GFP and pCAG-Sox11-IRES-GFP. For retrovirus-mediated expression of Neurog2, the corresponding cDNA was cloned into the pCAG-IRES-dsRed (Heinrich et al., 2010, 2011) to generate pCAG-Neurog2-IRES-dsRed. pCAG-GFP-IRES-Cre was generated from the pCAG-GFP vector (Zhao et al., 2006) by replacing the GFP coding sequence with cDNA for GFP and Cre recombinase as well as an internal ribosomal entry site (IRES).

Retroviruses were generated as described previously (Tashiro et al., 2006). Virus-containing supernatant was harvested four times every $48 \mathrm{~h}$ after transfection and concentrated by two rounds of ultracentrifugation. Viral titers were $\sim 5 \times 10^{8}$ colony-forming units $/ \mathrm{ml}$.

Stereotactic injections. Mice were deeply anesthetized with a mixture of ketamine ( $100 \mathrm{mg} / \mathrm{kg}$ bodyweight) and xylazine ( $10 \mathrm{mg} / \mathrm{kg}$ bodyweight). Mice were stereotactically injected with $1 \mu \mathrm{l}$ of the CAG-GFP or CAGGFP-IRES-Cre retroviruses into the left and right dentate gyrus (coordinates from bregma were $-1.9 \mathrm{~mm}$ anterior/posterior, $\pm 1.6 \mathrm{~mm}$ medial/lateral, $-1.9 \mathrm{~mm}$ dorsal/ventral from dura).

Tissue processing. Mice were killed using $\mathrm{CO}_{2}$. They were perfused with PBS, pH 7.4, for $5 \mathrm{~min}$, followed by $4 \%$ paraformaldehyde (PFA) for 5 min. Brains were removed and postfixed in $4 \%$ PFA for $12 \mathrm{~h}$ at $4^{\circ} \mathrm{C}$ and were subsequently transferred to a $30 \%$ sucrose solution. Fortymicrometer-thick coronal brain sections were cut using a sliding microtome (Leica Microsystems).

Histology and counting procedures. Sections were blocked in Trisbuffered saline (TBS) supplemented with 3\% normal donkey serum and $0.25 \%$ Triton X-100 for $60 \mathrm{~min}$. Brain sections were incubated in block- ing solution containing the primary antibodies at the appropriate dilutions at $4^{\circ} \mathrm{C}$ for $48 \mathrm{~h}$. Primary antibodies against the following antigens were used: Sox4 (guinea pig, 1:1000; Hoser et al., 2008), Sox11 (goat, 1:250; Santa Cruz Biotechnology), Sox11 (guinea pig, 1:500; Hoser et al., 2008), Sox2 (goat, 1:1000; Santa Cruz Biotechnology), Sox2 (rabbit, 1:1000; Millipore), GFAP (rabbit, 1:500; Dako), doublecortin (DCX) (goat, 1:250; Santa Cruz Biotechnology), calretinin (rabbit, 1:1000; Swant), calbindin (rabbit, 1:1000; Swant), NeuN (mouse, 1:50; Millipore), GFP (chicken, 1:1000; Aves Labs), RFP (rat, 1:50; Rottach et al., 2008), NeuroD1 (goat, 1:200; Santa Cruz Biotechnology), Tbr2 (rabbit, 1:500; Abcam), Ki67 (rabbit, 1:1000; Novocastra Laboratories), and activated caspase 3 (rabbit, 1:150; BD Biosciences). After three washes in TBS, samples were incubated in blocking solution containing the secondary antibody coupled to Cy3, Cy5, FITC, or Alexa Fluor 488 for $2 \mathrm{~h}$ at room temperature. Secondary antibodies were obtained from The Jackson Laboratory and were used at a dilution of 1:250 after resuspension in $200 \mu \mathrm{l}$ of $\mathrm{H}_{2} \mathrm{O}$ and $200 \mu \mathrm{l}$ of glycerol. Samples were washed three times with TBS, incubated in $10 \mathrm{mg} / \mathrm{ml} \mathrm{4}$, 6-diamidino-2-phenylindole (DAPI) (1:10,000; Sigma-Aldrich) for $10 \mathrm{~min}$, and mounted in Aqua PolyMount (Polysciences). To characterize the phenotype of the retrovirally transduced cells, equidistant sections containing the dentate gyrus were selected and stained. Transduced cells were identified based on the expression of GFP and were analyzed by confocal microscopy for immunoreactivity for the respective marker $(n>50$ cells per animal and marker). Coexpression of Sox 4 and Sox 11 with stage-specific markers was performed in at least three sections containing the dentate gyrus from three different animals. Confocal single-plane images and $Z$-stacks were taken on an Olympus FluoView 1000 or a Leica SP5 confocal microscope.

NSC culture. Neural stem/progenitor cells were isolated from the hippocampal dentate gyrus according to the protocol by Ray and Gage (2006) with the exception that whole-hippocampal tissue was used instead of whole-brain tissue. Cells were cultured on poly-D-lysine (PDL; $10 \mu \mathrm{g} / \mathrm{ml}$; Sigma) and laminin (5 $\mu \mathrm{g} / \mathrm{ml}$; Invitrogen) coated plates in DMEM/F-12 medium (Invitrogen) containing B27 supplement (Invitrogen), $8 \mathrm{~mm}$ HEPES buffer, $1 \times$ penicillin/streptomycin/fungizone (Invitrogen), $20 \mathrm{ng} / \mathrm{ml}$ epidermal growth factor (EGF; PeproTech), and 20 $\mathrm{ng} / \mathrm{ml}$ basic fibroblast growth factor (FGF2; PeproTech). Cultures were supplemented with growth factors every other day.

For deletion and overexpression experiments, $1.5 \times 10 \mathrm{e} 5$ cells per well were seeded on PDL/laminin-coated 24-well plates. For deletion experiments, cells were transduced with CAG-GFP or CAG-GFP-IRES-Cre retrovirus [multiplicity of infection (MOI) of $\sim 1$ ]. At $48 \mathrm{~h}$ after transduction, differentiation was induced by growth factor withdrawal. For overexpression experiments, cells were transduced with CAG-GFP, CAG-Sox4-IRES-GFP, or CAG-Sox11-IRES-GFP retrovirus (MOI of $\sim 1$ ). Cultures were fixed $6 \mathrm{~d}$ (deletion experiments) or $2 \mathrm{~d}$ (overexpression experiments) after transduction with 4\% PFA for $12 \mathrm{~min}$. For BrdU incorporation studies, cultures were treated $2 \mathrm{~d}$ after transduction with 5 $\mu \mathrm{M}$ BrdU for $1 \mathrm{~h}$ before fixation. Cells were processed for immunostaining, as described previously (Lie et al., 2005). Primary antibodies against the following antigens were used: BrdU (rat, 1:500; AbD Serotec/Morphosys), DCX (goat, 1:250; Santa Cruz Biotechnology), GFAP (mouse, 1:1000; Sigma-Aldrich), GFP (chicken, 1:500; Aves Labs), Sox2 (goat, 1:1000; Santa Cruz Biotechnology), and receptor interacting protein (RIP; mouse; Developmental Studies Hybridoma Bank). Single-plane images were taken on a Leica DMI 6000B fluorescence microscope. The percentage of marker-positive cells among the total GFP-positive population was determined for three wells within three different biological replicates. Approximately 1500 transduced cells from five to seven randomly selected fields were evaluated for each condition in each experiment.

Caspase 3 activity assay. Caspase activity was measured using the Caspase-Glo 3/7 Assay (Promega). Briefly, cells were lysed in 1\% (v/v) NP-40 and $0.1 \%(\mathrm{v} / \mathrm{v})$ SDS in PBS containing phosphatase and protease inhibitors (PhosphoSTOP and Complete EDTA-free, respectively; Roche). After $20 \mathrm{~min}$ on ice, cell lysates were centrifuged at $14,000 \times \mathrm{g}$ for $10 \mathrm{~min}$ at $4^{\circ} \mathrm{C}$. Supernatants were then collected for caspase activity measurements. Ten micrograms of protein of each sample in $50 \mu \mathrm{l}$ of 
lysis buffer were mixed with $50 \mu$ l Caspase-Glo 3/7 reagent in a 96-well plate and incubated under slow shaking for $30 \mathrm{~min}$. Luciferase activity was measured using a Luminometer Centro LB 960 (Berthold Technologies). Luminescence was proportional to the amount of caspase activity present in the samples. Results are the average of three biological replicates.

Reporter assays. HEK293 cells were transfected using the $\mathrm{CaCl}_{2}$ method with equal molar amounts (500 fmol) of the pCAG-GFP, pCAG-Sox4IRES-GFP, or pCAG-Sox11-IRES-GFP plasmids together with DCXpromoter Luciferase reporter constructs (120 ng) (Karl et al., 2005) and a Renilla-luciferase under the control of the human elongation factor 1 promoter (10 ng) (Lie et al., 2005). Cell harvest and analysis were performed $48 \mathrm{~h}$ after transfection using the Promega dual luciferase kit and a Centro LB 960 luminometer. Luciferase assays were performed from three biological replicates.

Mutations to the human DCX promoter were introduced using the QuikChange II XL Site-Directed Mutagenesis Kit (Agilent) according to the instructions of the manufacturer instructions. The following primers were used: 5' -CAA TTT TCT AAA AGA AAG GGC TAG CAC AGG GCT CAT TAG AGC ATT CTG ATT-3' and 5'-AAT CAG AAT GCT CTA ATG AGC CCT GTG CTA GCC CTT TCT TTT AGA AAA TTG-3'. Mutagenesis PCR resulted in two point mutations (i.e., CATTGCT to CAGGGCT, which corresponds to AGCAATG and AGCCCTG, respectively, on the minus strand) to the predicted SoxC binding site located between the putative CAAT and TATA elements at position -2037 to -2031 relative to ATG.

$R N A$ isolation and RT-qPCR. Total RNA was isolated using Trizol reagent (Invitrogen). Isolated RNA was treated with DNase (Promega) according to the protocol of the manufacturer. cDNA was synthesized using Random Hexamer primers (Fermentas), RiboLock RNase I (Fermentas), and the RevertAid Premium Reverse Transcriptase (Fermentas). Real-time qPCRs were performed on a StepOne device (Applied Biosystems). Power SYBR Green PCR Master Mix (Applied Biosystems) was used for detection. Primers for qPCR were as follows: Sox4 forward, 5'-GAA CGC CTT TAT GGT GTG GT-3' and reverse, 5'-GAA CGG AAT CTT GTC GCT GT-3'; Sox11 forward, 5' -CCC TGT CGC TGG TGG ATA AG-3' and reverse, 5' -GGT CGG AGA AGT TCG CCT C-3'; DCX forward, $5^{\prime}$-TGC TCA AGC CAG AGA GAA CA-3' and reverse, 5'-CTG CTT TCC ATC AAG GGT GT-3'; $\beta$-tubulin III forward, $5^{\prime}$ CTG GAA CCA TGG ACA GTG TTC G-3' and reverse, 5' -CGA CAT CTA GGA CTG AGT CCA C-3'; and GFAP forward, 5' -AAC GAC TAT CGC CGC CAA CTG C- $3^{\prime}$ and reverse, $5^{\prime}$-ATG GCG CTC TTC CTG TTC GC- $3^{\prime}$. qPCR experiments were performed with cDNA from three independent biological replicates.

Chromatin immunoprecipitations. Chromatin immunoprecipitation (ChIP) experiments were performed on formaldehyde-fixed cells as described previously (Ehm et al., 2010), using the antibodies anti-Sox11 (Santa Cruz Biotechnology) and anti-polymerase (Pol II) (Abcam). As a negative control, the extracts were incubated with preimmune normal rabbit serum (IgG). The immune complexes were collected by incubation with protein G Dynabeads (Invitrogen). DNA was purified from each reaction using the Chromatin IP DNA Purification Kit from Active Motif. For the qPCR, the Brilliant II Fast SYBR Green qPCR Master Mix (Agilent) was used according to the protocol of the manufacturer on the Applied Biosystems StepOnePlus Instrument. The amount of precipitated DNA relative to input was determined as percentage $(\mathrm{ChIP} /$ total input $)$, which equals $2[(\mathrm{Ct}(x \%$ input $)-\log (x \%) / \log 2)-\mathrm{Ct}(\mathrm{ChIP})] \times 100$, where $x$ is the dilution of the input (1:100). All results were normalized to unspecific $\operatorname{IgG}=1$. The sequences of the primers sets used for the ChIP reactions on the mouse DCX promoter were the following: amplicon $\mathrm{A}$, forward 5'-CAG CCT TCA TGA TTC CTG CAA C-3' and reverse, 5' CTT CTG CCT AGT GGC TGG ACT-3'; amplicon B forward, 5'-GGT TTT AGC CAA GTA AAA TTG CC- $3^{\prime}$ and reverse, $5^{\prime}$-CCT GTA ACT GAA GCT TAG CGA AC-3'; amplicon C forward, $5^{\prime}$-GTT CGC TAA GCT TCA GTT ACA GG-3' and reverse, 5' ${ }^{\prime}$-AAG AAA GCC GTG TGC CTT GTG C-3'; and amplicon D forward, 5' -GCA CAC GGC TTT CTT TCT CT- $3^{\prime}$ and reverse, $5^{\prime}$-GTA AGA GCC CAC CTC CTC AA- $3^{\prime}$.

Primary cultures from postnatal cortical astroglia. For culturing postnatal astroglia, we followed the procedure described previously by Heinrich et al. (2010, 2011). After removal of the meninges, gray matter tissue from postnatal day 5 (P5) to P7 cerebral cortex of C57BL/6J or Sox4/ $11 \mathrm{dcKO}$ homozygote or heterozygote mice was dissected and dissociated mechanically. Cells were centrifuged for $5 \mathrm{~min}$ at $1000 \mathrm{rpm}$, resuspended, and plated in a medium consisting of DMEM/F-12 (Invitrogen), $3.5 \mathrm{~mm}$ glucose (Sigma), 10\% fetal calf serum (Invitrogen), penicillin/ streptomycin (Invitrogen), and supplemented with B27 (Invitrogen), $10 \mathrm{ng} / \mathrm{ml}$ EGF (Roche), and $10 \mathrm{ng} / \mathrm{ml}$ FGF2 (Roche). Cells were passaged after 1 week using trypsin/EDTA (Invitrogen) and plated on PDL (SigmaAldrich) glass coated coverslips at a density of 50,000 cells per coverslip (in 24-well plates; BD Biosciences) in the same medium.

Astroglia reprogramming assays. Two to four hours after plating into PDL-coated coverslips, astroglia cells were infected using vesicular stomatitis virus glycoprotein-pseudotyped retroviruses encoding various genes. Twenty-four hours after transduction, the medium of astroglia was replaced by N3 differentiation medium, consisting of DMEM/F-12, $25 \mu \mathrm{g} / \mathrm{ml}$ insulin, $50 \mu \mathrm{g} / \mathrm{ml}$ transferrin, $30 \mathrm{~nm}$ sodium selenite, $20 \mathrm{~nm}$ progesterone (Sigma), $100 \mathrm{~nm}$ putrescine (Sigma), and penicillin/streptomycin. The cells were allowed for differentiation $7 \mathrm{~d}$ and then fixed in 4\% PFA in PBS for 15 min for subsequent analysis. Cells were first incubated in blocking solution (0.5\% Triton X-100 and 3\% BSA in PBS) for $30 \mathrm{~min}$. Primary antibodies were incubated on specimens for $2 \mathrm{~h}$ at room temperature in blocking solution. The following primary antibodies were used: GFP (chicken, 1:300; Aves Labs), RFP (rat, 1:300; Chromotek), $\beta$-tubulin III (mouse, 1:500; Sigma), and DCX (goat, 1:250; Santa Cruz Biotechnology). After washing in PBS, cells were incubated with appropriate species- or subclass-specific secondary antibodies conjugated to Сy2, Сy3, Сy5 (1:1000; Jackson ImmunoResearch), Alexa Fluor-488 (1: 1000; Invitrogen), and TRITC (1:1000; Jackson ImmunoResearch) for $2 \mathrm{~h}$ at room temperature, followed by extensive washing in PBS. Coverslips were finally mounted onto a glass slide with an antifading mounting medium (Aqua Poly/Mount; Polysciences). Stainings were analyzed with laser-scanning confocal microscope (LSM710; Carl Zeiss). Digital images were captured using ZEN software (Carl Zeiss). Cell counts were performed by taking pictures of several randomly selected views per coverslip analyzed by means of a Carl Zeiss LSM 710 confocal microscope using a $25 \times$ objective. Subsequently, pictures were analyzed for cell quantification using NIH Image J 1.42q software. Three to seven independent experiments per each assay were performed.

Statistical analysis. Unpaired Student's $t$ test was used for analysis. Differences were considered statistically significant at ${ }^{\star} p<0.05,{ }^{*} p<$ 0.01 , and ${ }^{* * *} p<0.001$. All data are presented as mean \pm SEM.

\section{Results \\ Overlapping expression of Sox 4 and Sox 11 in the adult hippocampal neurogenic lineage}

Our previous analysis demonstrated that Sox 11 protein is almost exclusively expressed in immature neurons in the adult neurogenic areas (Haslinger et al., 2009). In the developing CNS, the Sox 11 homolog Sox 4 is frequently coexpressed and exerts functions redundant to Sox 11. Immunostaining using a Sox4-specific antiserum (Potzner et al., 2007) revealed that Sox 4 expression is primarily confined to areas of adult neurogenesis, i.e., the hippocampal dentate gyrus (Fig. $1 A$ ), the subventricular zone of the lateral ventricles (Fig. $1 B$ ), the rostral migratory stream, and the olfactory bulb (Fig. 1C). In the following, we focused on the function of Sox 4 and Sox 11 transcription factors in the regulation of adult hippocampal neurogenesis.

First, we established the precise expression pattern of Sox4/ Sox11 in the adult hippocampal neurogenic lineage. Sox 4 expression showed almost complete overlap with the expression of Sox11 in the adult hippocampal neurogenic niche ( $>98 \%$ of Sox $4^{+}$cells coexpressed Sox $11^{+}$and vice versa) (Fig. 2A). Detailed analysis using stage-specific markers for adult hippocampal neurogenesis showed that Sox 4 and Sox 11 were only occasionally coexpressed with the SoxB1 transcription factor

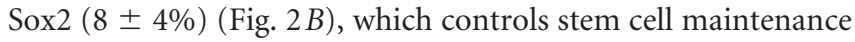



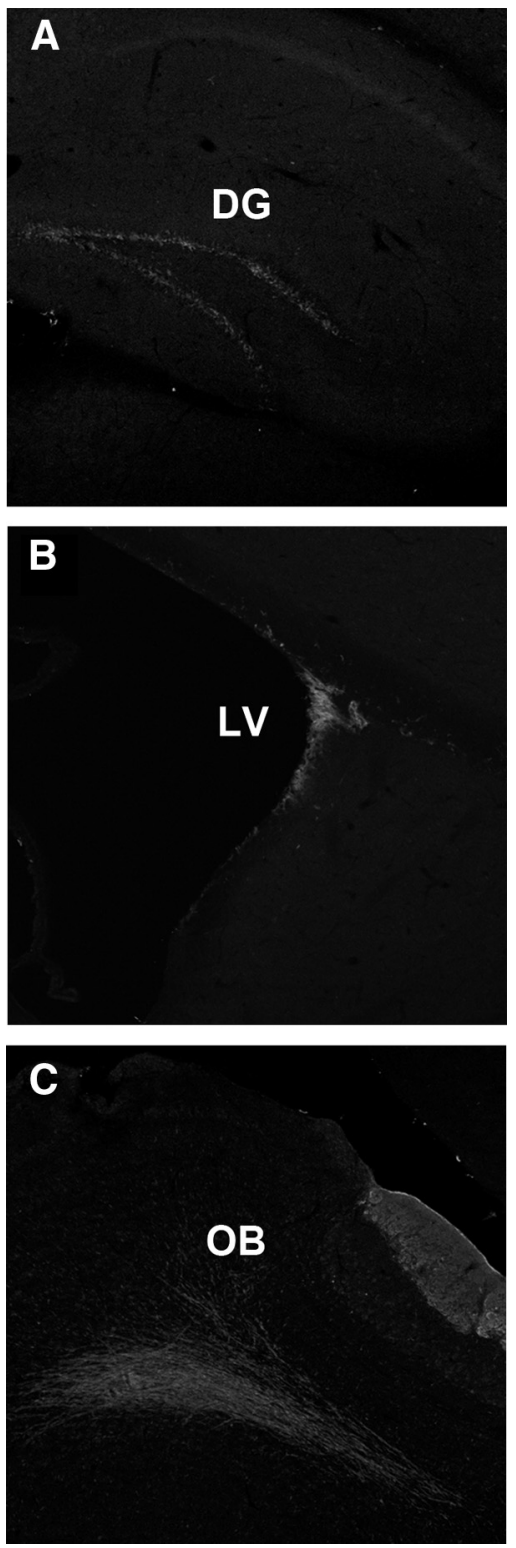

Figure 1. Sox4 transcription factor expression in the adult neurogenic niches. Sox4 (gray) is highly expressed in the dentate gyrus $(D G)(A)$, the subventricular zone of the lateral ventricle $(\mathrm{LV})(\boldsymbol{B})$, and the rostral migratory stream of the olfactory bulb (OB) (C). Additional expression is found in the olfactory tubercle.

and proliferation (Pevny and Placzek, 2005; Favaro et al., 2009; Ehm et al., 2010) and is expressed by Type 1 and Type 2a/b stem/progenitor cells in the adult hippocampus (Steiner et al., 2006; Suh et al., 2007). A significant fraction of the Sox4- and Sox11-positive cells were positive for the transcription factor Tbr2 (19 $\pm 2 \%$ ) (Fig. 2C), which is expressed by Type $2 \mathrm{a} / \mathrm{b}$ and Type 3 intermediate progenitor cells (Hodge et al., 2008). Consistent with their expression in proliferating progenitor cells, a subset of Sox4- and Sox11-positive cells colabeled for the proliferation marker Ki67 ( $8 \pm 6 \%$ ) (Fig. $2 E-G$ ). Costaining with DCX and Ki67 further demonstrated that Sox4 and Sox11 are expressed neuronally committed precursor cells (Fig. $2 F, G$ ). Similar to Sox11 (Haslinger et al., 2009), we found Sox4 to be strongly coexpressed with the immature neuronal marker DCX (99 \pm $1 \%$ ), and we only occasionally observed DCX-positive immature neurons that were negative for Sox 4 or Sox $11(<1 \%)$ (Haslinger et al., 2009). Moreover, SoxC proteins were strongly coexpressed with the transcription factors NeuroD1 $(94 \pm 1 \%)$ and Prox1 $(91 \pm 4 \%)($ Fig. $2 H)$, whereas almost no overlap was observed for Sox 4 (3 $\pm 2 \%$ ) and Sox11 (Haslinger et al., 2009) with the mature dentate granule cell marker Calbindin (Fig. 2D). Together, these data indicate that expression of the SoxC proteins Sox 4 and Sox11 is likely to be initiated around the time of neuronal fate commitment of hippocampal neural progenitors, i.e., in late Type $2 \mathrm{a}$ and Type $2 \mathrm{~b}$ cells, is maintained in immature neurons and is finally downregulated during maturation (Fig. 2I).

\section{SoxC transcription factors are essential for in vitro neurogenesis from adult neural precursor cells}

The timing of the initiation of Sox 4 and Sox 11 protein expression raised the possibility that SoxC transcription factors participate in the control of neuronal fate determination in adult hippocampal neurogenesis. To test this hypothesis, we first examined the consequences of Sox4/Sox 11 ablation on differentiation in vitro. NSCs were isolated from the hippocampus of adult Sox $4^{\text {flox/flox }}$; Sox $11^{\text {flox/flox }}$ double conditional knock-out mice (Sox4/Sox11dcKO). Recombination of the floxed loci was induced by transduction with a retrovirus bicistronically encoding for Cre recombinase and GFP (CAG-GFP-IRES-Cre); a GFP-expressing retrovirus served as control. Transduced cultures were differentiated by growth factor withdrawal and were analyzed 6 d later. Transduction efficiency reached $\sim 70-80 \%$ for both retroviruses. Sox 4 and Sox 11 mRNAs were strongly reduced in CAG-GFP-IRES-Cre transduced cultures (Fig. 3B), indicating that Sox4 and Sox11 loci were efficiently recombined by the retrovirally expressed Cre recombinase. Sox4/Sox11 ablation consistently resulted in decreased cell densities, raising the possibility that these proteins are involved in the regulation of survival and proliferation (Fig. 3A). No difference in cell death as determined by measurement of caspase 3 activity was found (Fig. $3 D$ ). The fraction of BrdU-labeled cells after a $1 \mathrm{~h} \mathrm{BrdU}$ pulse before analysis, however, was significantly reduced among Sox4/Sox11deficient cells $(11.9 \pm 1.8 \%$ in control; $6.0 \pm 1.0 \%$; Fig. $3 C$ ), suggesting that Sox4/Sox11 ablation resulted in decreased proliferation of differentiating adult NSCs. Compared with controls, Sox4/Sox11-deficient cells only rarely displayed a neuronal morphology but instead had large nuclei and cell bodies with thick processes (Fig. $3 A$ ), raising the possibility that neurogenesis from Sox4/Sox11-deficient NSCs was impaired. Indeed, expression of the neuron-specific genes DCX and $\beta$-tubulin III was strongly decreased in Cre-transduced cultures (Fig. 3B). Moreover, Sox4/ Sox 11 -deficient cells showed an $\sim 90 \%$ decrease in the generation of DCX-positive neurons $(9.4 \pm 0.9 \%$ among control transduced cells; $0.8 \pm 0.3 \%$ among Cre transduced cells; Fig. $3 A$ ) and showed a trend toward higher numbers of Sox2-immunoreactive cells $(14.1 \pm 5.5 \%$ among control transduced cells; $26.8 \pm 7.5 \%$ among Cre transduced cells). Importantly, Sox4/Sox11-deficient NSCs produced GFAP-positive astrocytes at a frequency comparable with control (Fig. 3A,B), indicating that Sox4/Sox11 are essential for neurogenesis but not for astrogliogenesis from adult NSCs.

To further test the function of SoxC proteins in neurogenesis, we performed gain-of-function experiments in adult hippocampus-derived NSC cultures. NSCs were transduced with retroviruses bicistronically encoding for GFP and either Sox11 (CAG-Sox11-IRES-GFP) or Sox4 (CAG-Sox4-IRES-GFP). NSCs transduced with retroviruses encoding for GFP (CAGGFP) served as controls. Transduction efficiencies reached 

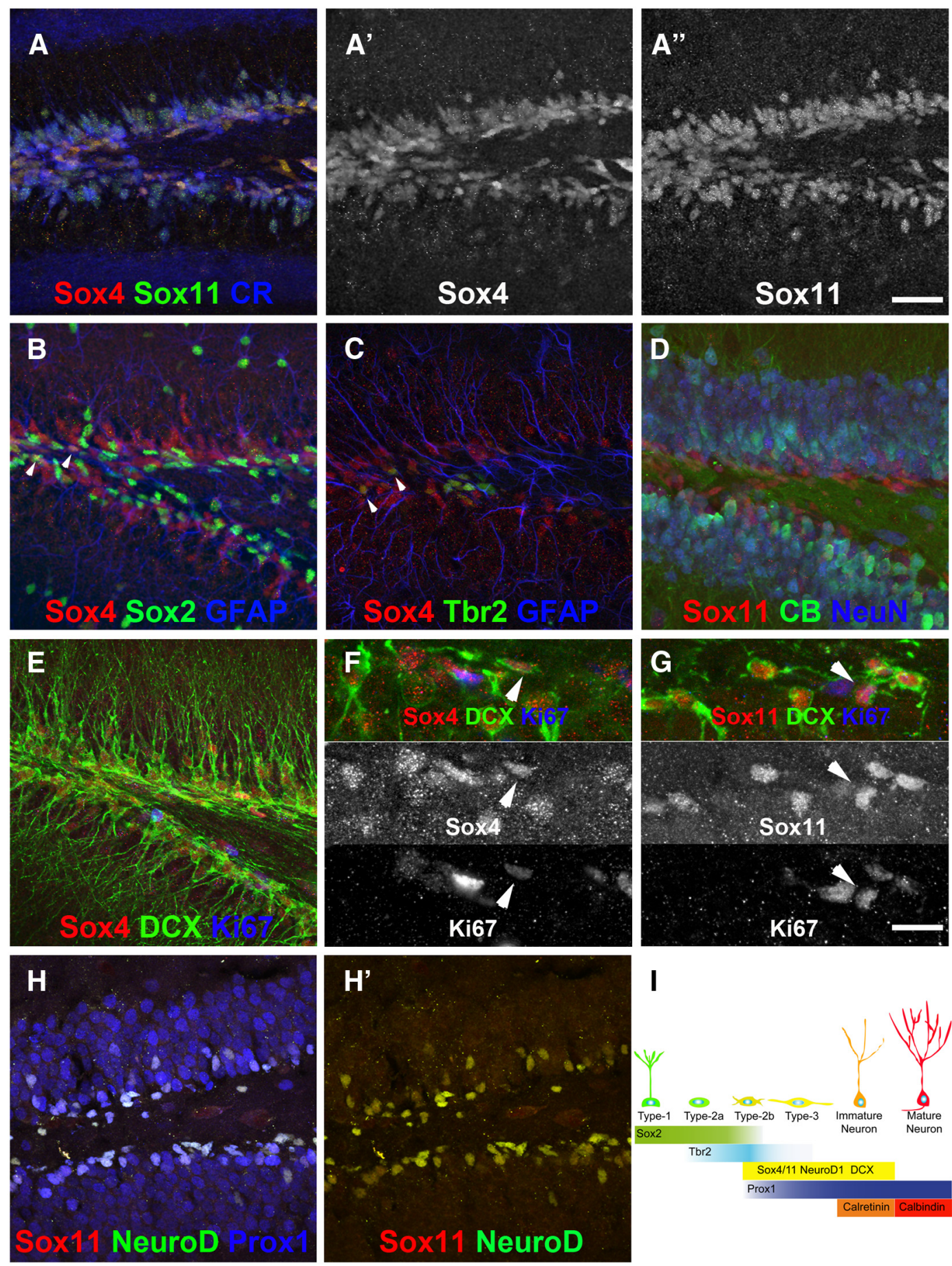

Figure 2. SoxC transcription factor expression in the adult hippocampal neurogenic lineage. $A$, Sox4 (red) and Sox 11 (green) are coexpressed in the dentate gyrus. Sox4/Sox 11 double-positive cells appear in yellow. Multiple cells are also positive for calretinin (blue), which is transiently expressed by postmitotic immature dentate granule neurons (Brandt et al., 2003). Single channels for Sox4 and Sox11 are presented in $\left(\boldsymbol{A}^{\prime}, \boldsymbol{A}^{\prime \prime}\right)$. B, Sox4 (red) is rarely expressed in Sox2 (green) expressing precursor cells (arrowheads). GFAP in blue. C, A fraction of Tbr2-positive precursor cells (green) expresses Sox4 (red). GFAP in blue. D, Mature calbindin-positive (CB, green) dentate granule cells are primarily negative for Sox11 (red). NeuN in blue. $\boldsymbol{E}$, Sox4 (red) and DCX (green) show almost completely overlapping expression patterns. F, Sox4 (red) is expressed in DCX (green) and Ki67 (blue) positive proliferating precursors (arrowheads). G, Sox 11 (red) is expressed in DCX (green) and Ki67 (blue) positive proliferating precursors (arrowheads). $\boldsymbol{H}, \boldsymbol{H}^{\prime}$, Sox 11 (red) is coexpressed with NeuroD1 (green) and Prox1 (blue). Triple-labeled cells in $\boldsymbol{H}$ appear in white and double-labeled cells in $H^{\prime}$ in yellow. $I$, Schematic drawing showing a model of adult hippocampal neurogenesis and Sox4/Sox11 expression in relation to expression of other markers along the cell lineage of the dentate gyrus. Scale bars: $\boldsymbol{A}-\boldsymbol{E}, \boldsymbol{H}, 40 \mu \mathrm{m} ; \boldsymbol{F}, \mathbf{G}, 20 \mu \mathrm{m}$.

$\sim 70 \%$ for the Sox 11 retrovirus and $50-55 \%$ for the Sox 4 retrovirus. After transduction, the cells were kept for an additional $2 \mathrm{~d}$ in proliferating culture conditions, i.e., in the presence of EGF and FGF2. RT-qPCR verified overexpression of the transgene (data not shown). Strikingly, Sox4 and Sox 11 overexpression en- hanced expression of mRNAs for the immature neuronal structural protein DCX and $\beta$-tubulin III (Fig. $4 C$ ) and the generation of DCX-expressing cells with immature neuronal morphology among the transduced cells $(2.3 \pm 0.2 \%$ in control; $9.8 \pm 2.3 \%$ among Sox 4 transduced cells; $16.1 \pm 1.7 \%$ among Sox 11 trans- 
duced cells; Fig. 4A) despite the proliferation-promoting culture conditions. No significant changes were observed with regard to caspase 3 activity (Fig. $4 E$ ), indicating that SoxC proteins did not enhance neuronal survival. Neither Sox4 nor Sox11 overexpression altered BrdU incorporation among transduced cells (Fig. 4D), arguing against the possibility that SoxC proteins increase neurogenesis through promoting proliferation of DCX-positive neuroblasts. Importantly, overexpression of Sox 4 and Sox11 did not induce the generation of astrocytes, which were identified by the astroglia-specific protein GFAP (Fig. 4B), and we almost never observed RIP-positive oligodendroglial cells $(<0.1 \%$ in all conditions). These observations strongly suggest that SoxC proteins specifically promote neurogenesis, which-although not formally tested-may occur at the expense of NSCs.

Together, the results of the in vitro gain- and loss-of-function experiments indicate an essential role for Sox 4 and Sox11 in the control of neuronal differentiation of adult NSCs. The fact that SoxC proteins induce the generation of DCXexpressing neurons even under culture conditions that favor the expansion of stem cells further illustrates the potency of these transcription factors to activate a neuronal differentiation program.

\section{In vivo conditional ablation of} Sox4/Sox11 inhibits neurogenesis

Next, we ablated Sox4 and Sox11 in the adult hippocampal neurogenic lineage to determine their role in the in vivo context. Genetic ablation of Sox 4 and Sox 11 in individual proliferating precursors was achieved by stereotactic injection of the MMLV retrovirus CAG-GFP-IRES-Cre (Tashiro et al., 2006; Jagasia et al., 2009) into the dentate gyrus of adult Sox4/Sox11dcKO mice. Sox4/Sox11dcKO mice injected with GFP-encoding retrovirus served as controls. Animals were analyzed $5 \mathrm{~d}$ after retroviral injection. The majority of control retrovirus transduced cells expressed Sox 4 and Sox11 (>80\%). In contrast, CAG-GFP-IRES-Cre transduced cells did not express Sox 4 and Sox11, demonstrating efficient recombination of the floxed Sox 4 and Sox 11 alleles (Fig. $5 A^{\prime}, B^{\prime}$ ). No differences in the number of transduced cells were observed between the experimental groups. SoxC-ablated cells appeared healthy and did not display signs of apoptotic cell death, such as membrane blebbing or pyknotic nuclei. In addition, SoxC ablation did not alter the fraction of activated caspase 3 -positive cells $(<1 \%$ of transduced cells among control and SoxC-ablated cells) (Fig. 5F). Collectively, these observations indicate that SoxC deficiency did not affect survival at this time point.

Consistent with previous studies, control cells displayed a polarized morphology, bore neurites that paralleled the granule cell layer, and in some cases had already formed a primary dendrite that extended toward the molecular layer (Zhao et al., 2006). Moreover, expression of the early neuronal marker DCX (71.0 \pm $16.2 \%$ among transduced cells) and the dentate granule neuron- specific transcription factors NeuroD1 $(88.7 \pm 10.0 \%)$ and Prox1 $(33.9 \pm 16.7 \%)$ indicated that the majority of transduced cells had differentiated into neurons and had adopted a dentate granule neuron identity (Fig. 5A-C). In contrast, most Sox4- and Sox11-negative cells failed to develop a neuron-like polarized morphology and frequently displayed multiple short processes or a star-like morphology (Fig. $5 A^{\prime}-D^{\prime}, E-G$ ). In addition, Sox4/ Sox11-ablated newborn cells showed strongly impaired expression of neuron-specific markers. Virtually all Sox4/Sox11ablated newborn cells were DCX negative $(0.7 \pm 1.1 \%)$ (Fig. $\left.5 A^{\prime}, H\right)$ and showed reduced expression of NeuroD1 (17.8 \pm 8.9\%) (Fig. 5 $\left.B^{\prime}, H\right)$ and Proxl $\left(18.7 \pm 10.2 \%\right.$ ) (Fig. $\left.5 C^{\prime}, H\right)$. These findings indicate that Sox4/11-deficient cells failed to initiate or maintain a full neuronal differentiation program.

Sox4/Sox11-deficient cells were also analyzed for the expression of the stem/early progenitor markers Sox 2 and Tbr2, the proliferation marker Ki67, and the astroglial marker GFAP. Strikingly, many Sox4/Sox11-deficient cells expressed Sox2 (62.0 \pm 8.7 vs $30.0 \pm 8.2 \%$ in controls) (Fig. $5 C^{\prime}, E, E^{\prime}, H$ ). However, no differences were detected in the expression of Tbr2 (10.3 $\pm 4.2 \mathrm{vs}$ $14.3 \pm 10.4 \%$ among control and SoxC-ablated cells, respectively) (Fig. $5 D, D^{\prime}, H$ ) and the fraction of Ki67-positive cells among the retrovirally transduced cells $(4.6 \pm 2.3$ vs $6.8 \pm 4.9 \%)$ (Fig. 5G,H), ruling out the possibility that Sox4/Sox11-deficient 
A

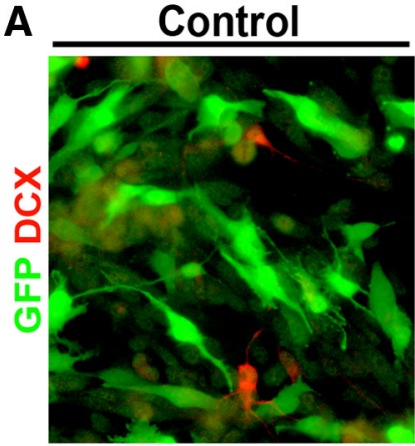

B

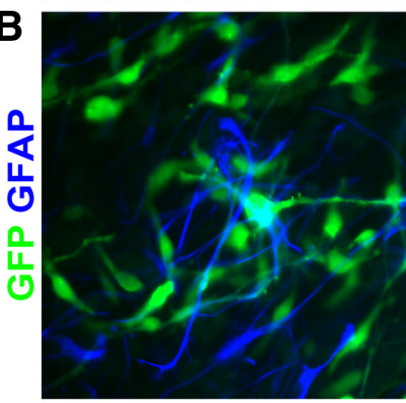

C

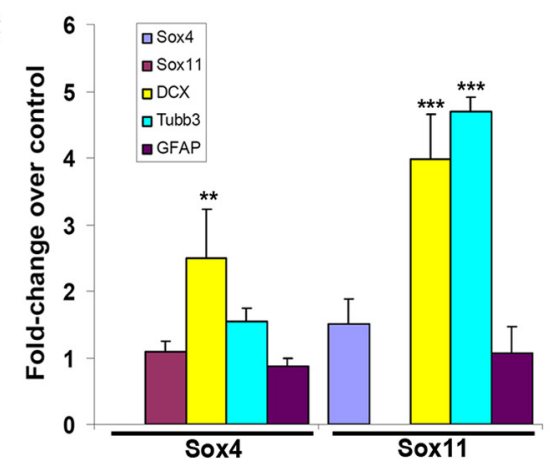

Sox4
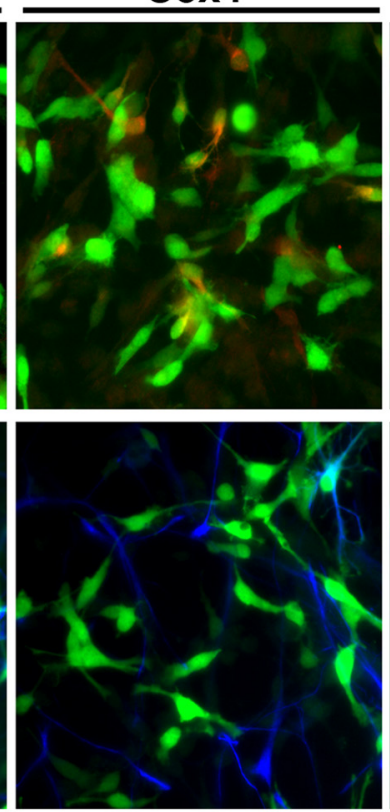

D
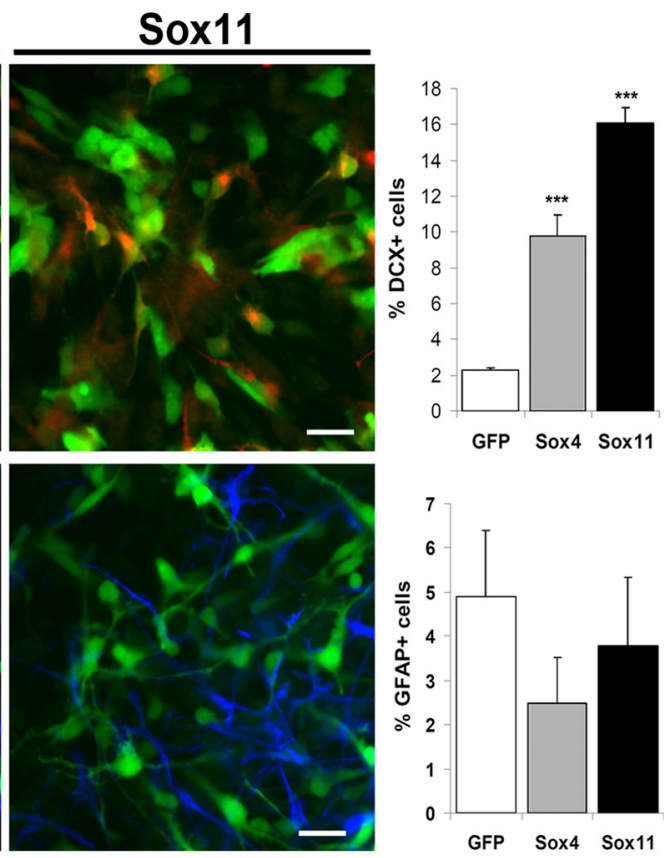

$\mathbf{E}$
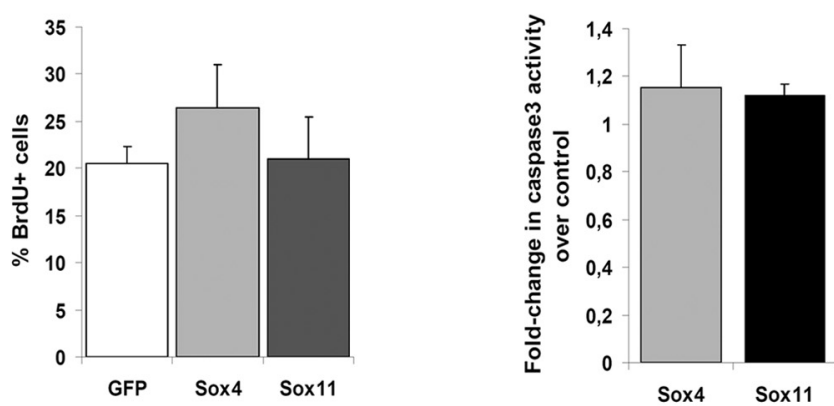

Figure 4. SoxC factors enhance in vitro neurogenesis from adult NSCS. $A$, Sox4 (middle) and Sox11 (right) overexpressing NSCs generate more DCX-positive (red) neurons. Transduced cells are identified by GFP expression (green). B, Sox4 (middle) and Sox11 (right) overexpression does not alter the generation of GFAP-positive (blue) astrocytes. Scale bar, $30 \mu \mathrm{m}$. C, qPCR analysis of Sox4 and Sox 11 transduced cultures. Sox11 transduced cultures express significantly higher levels of DCX and $\beta$-tubulin III (Tubb3) mRNA. Sox 4 overexpressing cultures express significantly higher levels of DCX and show a trend toward higher levels of $\beta$-tubulin III. The expression of GFAP is not affected. D, Sox 4 and Sox11 transduced NSCs do not incorporate BrdU to a higher percentage. E, Sox 4 and Sox11 overexpression do not alter caspase 3 activity. Scale bar, $30 \mu \mathrm{m}$.

cells remained as proliferative Sox2/Tbr2-positive Type 2a/b cells. Moreover, expression of GFAP remained unchanged, indicating that Sox4/Sox 11 deficiency did not promote astrocytic differentiation or adoption of a Sox $2^{+} / \mathrm{GFAP}^{+}$Type 1 cell identity (Fig. $5 E, H$ ). Thus, most Sox4/Sox11-deficient cells adopt a nonproliferative, GFAP ${ }^{-}$, Sox ${ }^{+}$identity. Whether these cells are postmitotic or retain the potential to proliferate and to differentiate, e.g., to glia, remains to be determined. Together, these data demonstrate that Sox4/Sox1 1 are required for neuronal differentiation of precursors in the adult hippocampal neurogenic lineage.

\section{SoxC proteins directly control expression of neuron-specific} proteins in adult NSCs

Results from our loss- and gain-of-function experiments strongly indicate that Sox 4 and Sox 11 are essential regulators of neuronal differentiation in adult hippocampal neurogenesis and predict that these factors are directly involved in the transcriptional control of neuron-specific genes in differentiating NSCs. The microtubule-associated protein DCX is specifically expressed in newly generated immature neurons of the adult brain and is con- sidered one of the earliest markers for neuronal lineage commitment in the adult neurogenic niches. Our current data also demonstrate that Sox 4 and Sox 11 expression is highly overlapping with the expression of DCX (Fig. 2 E; Haslinger et al., 2009). We therefore focused on the regulation of the DCX gene promoter to investigate potential direct effects of Sox4/Sox11 on neuronal lineage-specific gene expression. Previous analysis of the human DCX gene identified a $3.5 \mathrm{~kb}$ genomic region upstream of the DCX start codon (Karl et al., 2005) that faithfully reproduces activity of the endogenous DCX gene and targets transgene expression specifically to neuronal precursors and immature neurons in adult transgenic mice (Couillard-Despres et al., 2006, 2008; Zhang et al., 2010). Hence, this genomic fragment (hereafter named DCX promoter) contains key regulatory sequences for control of the DCX gene in the adult CNS. In silico analysis predicted several putative Sox binding sites (Harley et al., 1994) in this region. Indeed, reporter assays using a DCXpromoter-controlled luciferase reporter (Karl et al., 2005) revealed potent activation of the DCX promoter by Sox 4 and Sox 11 (Fig. 6A). In contrast, the stem-cell-associated transcription factor Sox 2 did not induce DCX promoter activity (Fig. 6A), indi- 

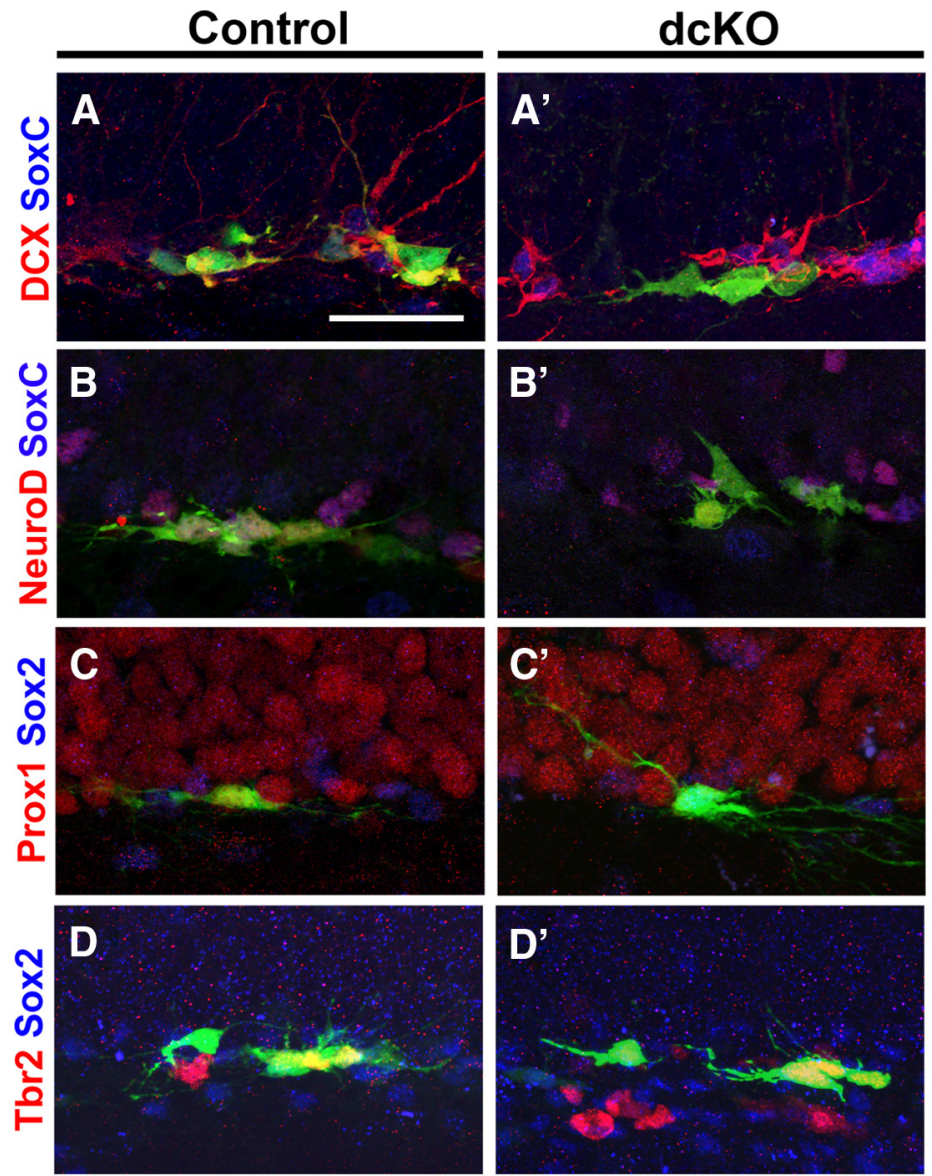

dcKO
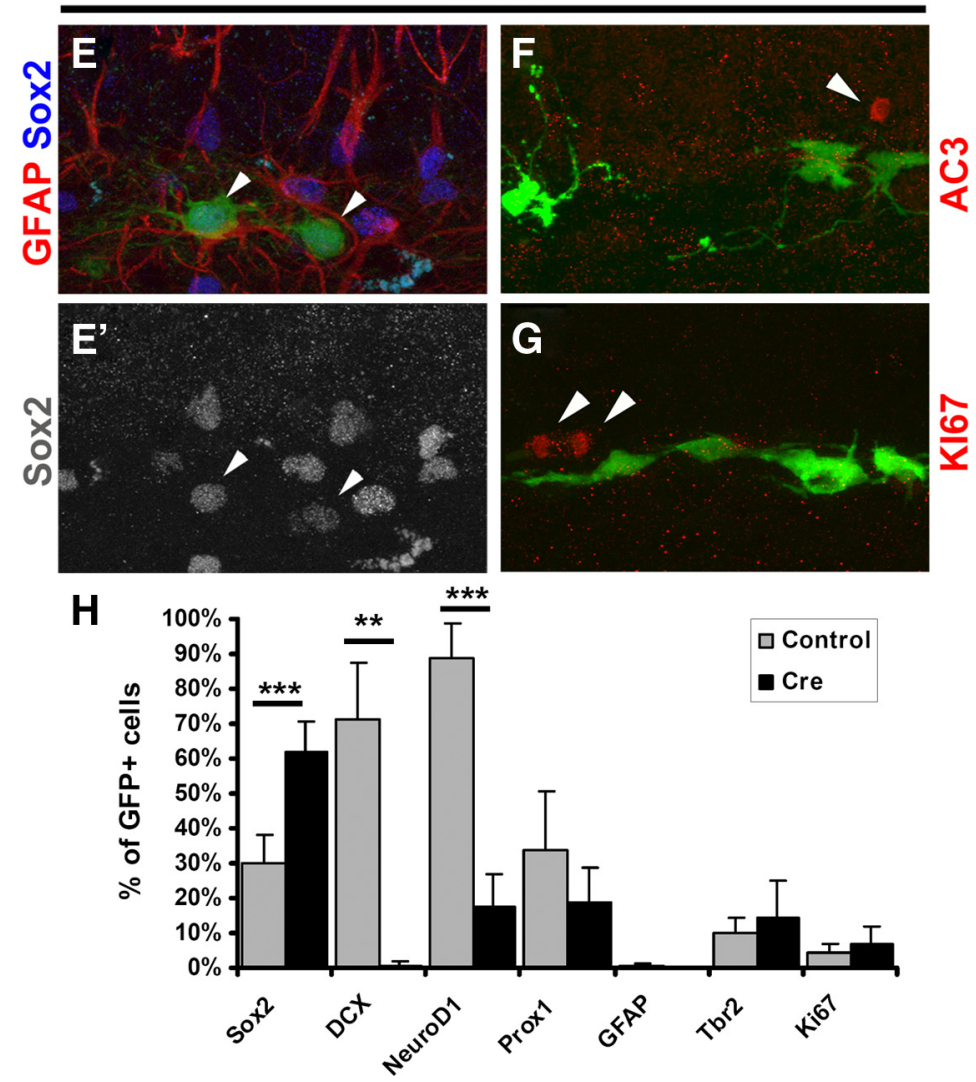

Figure 5. SoxC factors are essential for adult hippocampal neurogenesis. $\boldsymbol{A}-\boldsymbol{D}$, Left column shows transduced cells from dcKO mice injected with the control vector, and the right column shows transduced cells from dcKO mice injected with Cre recombinase. cating that, despite the fact that all Sox proteins bind to a common consensus motif in DNA (Harley et al., 1994), DCX promoter activation is a specific property of Sox 4 and Sox11. To identify potential Sox11-responsive areas, we used a series of deletion constructs used previously to define relevant regions for DCX promoter activity (Karl et al., 2005). As shown in Figure $6 B$, removal of the region between -2081 and -700 relative to the ATG start codon (mutant I) reduced dramatically the induction of the DCX promoter by Sox11. In contrast, mutants II $(-1793 /$ $-1388)$ and III $(-1386 /-784)$ retained most of the response to Sox11, suggesting that the region that is around or upstream of the major transcription start sites (TSS) might be responsible for the transactivation of the DCX promoter by Sox 11. Consistently, mutant IV, which lacks more distal $(-3509 /-2344)$ as well as proximal $(-1459 /-431)$ sequences, also exhibited a significant response to Sox 11 . We therefore considered the DCX promoter area between -2344 and -1388 as highly important for the transactivation by Sox 11 . A comparative analysis of the human and mouse sequences corresponding to this region revealed several potential Sox binding sites, the relative position and sequence of which are conserved between the two species (Fig. 6D). To test whether Sox11 directly transactivates DCX in vivo, we performed ChIP analysis and monitored four contiguous genomic regions on the mouse DCX promoter (amplicons A-D), scanning the Sox11-responsive region identified above. Because the avail-

\section{$\leftarrow$}

Transduced cells were identified by expression of GFP (green). SoxC expression (blue in $\boldsymbol{A}, \boldsymbol{B}$ ) was detected using Sox4 and Sox 11 antibodies generated in the same species. SoxC expression is not detected in Cre recombinase transduced cells. $\boldsymbol{A}$, Control cells were frequently positive for DCX (red). Moreover, some control cells had developed a primary dendrite that extended toward the molecular cell layer. $\boldsymbol{A}^{\prime}$, Sox4/Sox11ablated cells did not express DCX. In contrast to control cells, most Sox4/Sox11-ablated cells did not express NeuroD1 (red, $\boldsymbol{B}, \boldsymbol{B}^{\prime}$ ) or Prox1 (red, $\left.\boldsymbol{C}, \boldsymbol{C}^{\prime}\right)$. Control cells (D) and Sox4/Sox11ablated cells $\left(\boldsymbol{D}^{\prime}\right)$ expressed Tbr2 (red) at comparable frequencies. In both cases, Tbr2 ${ }^{+}$cells were mainly found in clusters. Sox2 in blue. Sox4/Sox11-ablated cells frequently expressed Sox2 (blue, $\boldsymbol{C}^{\prime}, \boldsymbol{E}, \boldsymbol{E}^{\prime}$ ) but were negative for GFAP $(\boldsymbol{E})$. Arrowheads in $\boldsymbol{E}$ and $\boldsymbol{E}^{\prime}$ point to Sox2-expressing Cre-transduced cells. Scale bar, $30 \mu \mathrm{m}$. $\boldsymbol{F}$, Sox4/Sox11 ablation did not increase apoptotic cell death. Representative image showing an activated caspase 3 ( $A C 3$; in red, arrowhead) cell and several AC3 negative, Cre-transduced cells. G, Sox4/Sox11-ablated cells were mostly postmitotic. Representative image showing $\mathrm{Ki}^{+}{ }^{+}$cells (in red, arrowheads) and several Ki67-negative, Cre-transduced cells. $\boldsymbol{H}$, Quantification of marker expression by transduced cells. 
A

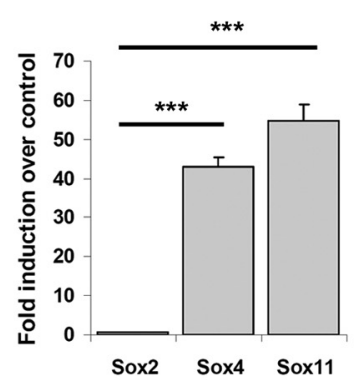

B

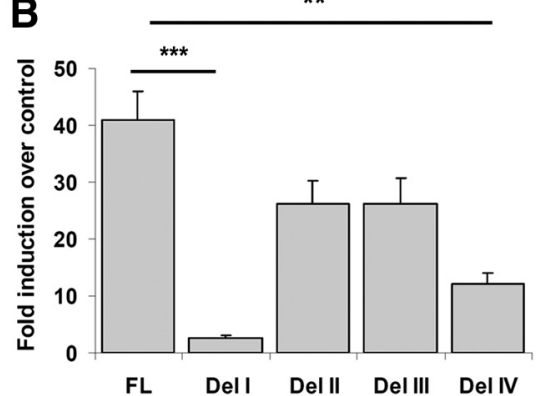

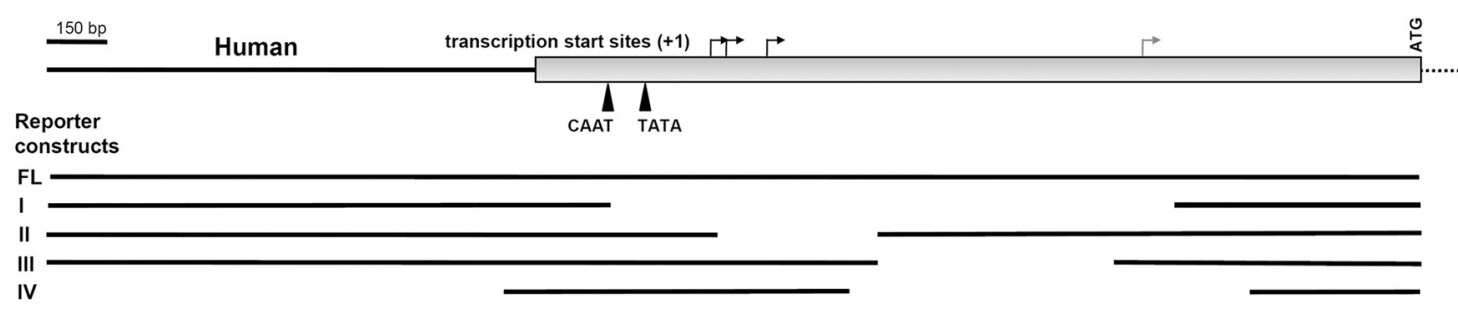

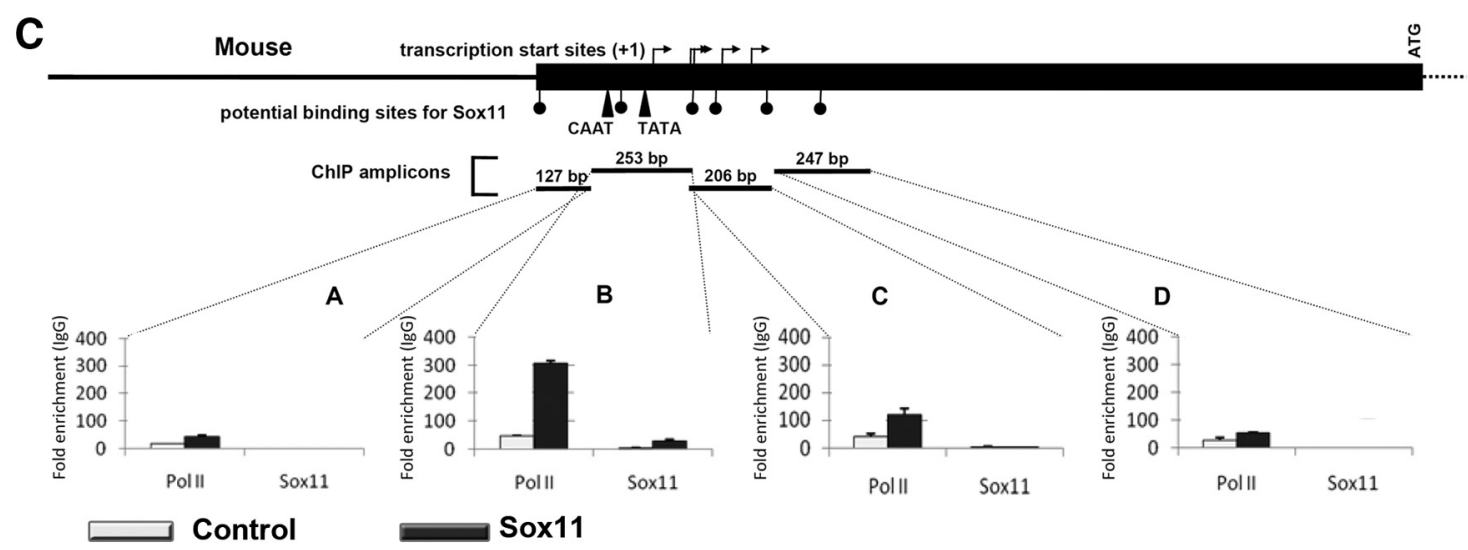

Control

Sox11

D Human 1 CTTTTTACTTCTATCTTCCATAATATTCTAAAATTATTTTCCCTTGTTTTGTTCCTATCCTACTTCCTCTTAGTCTACTTTGTTGACTTCATTAAAAAAC

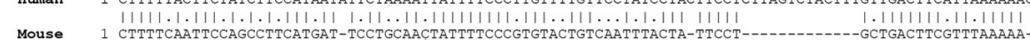
101 AAAAAACCAGTTGTTGGATACTTGAGCTAAACTGCCTTAAAGAATCTGCAGATTTTATTTTATTTTTTTTCTCTCAAGAGGGTAAAAGGAAGAGAGCTAC

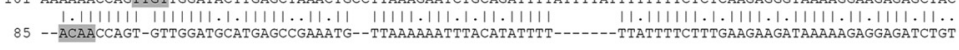

201 AATTTCTAAGAAGCCTGGCTTGGCTGTCTGAGTCTGGCCCCCAGGCAGATTAGGCCAAGGTTTTGGCCAAGTGAAATT GCCAATTTTTCTAAAAGAAAGGG

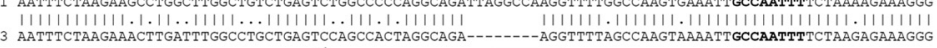
$\underset{\text { TATA }}{\longrightarrow}$

301 CTAGCACATTGCTCATTAGAGCATTCTGATTTTGTCTGCGCAATCTTTTTG---CTACCCCGCAATTTCCTGTTGGTTATAAATGAAACCTTTCTAGCTG

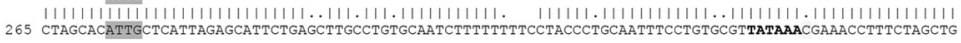
398 TTAATGCAGCCTGTGAATTTTTTTAAAA---GCATGTAATTAATCATAGGAGGTTGGGGGGATTCACTAAGCCTGAGTTACATGGGAGAAGCTGGACAÄ 365 TTAATGCAGGCTGTGAATTGA...1111 $111111111111111111111111111 \ldots 111.11111 .1 .1111111 .1111111111111111$ 495 GCACTAGGACCTAGAAGGCATCTATCCACCCTGGCAGGAATTTCTTGCTTGGAGCTCAGACAACAAAGGCATAGAGAGATTGGTTTCTTTCTCTCAGCA 465 GCACTAGGACCTAGAaGGCAACTATCCACCCTGGCAGGAATTTCTTGCTTGGAGCTCAGACAACAAAGGCATAGAGAGATTGGTTTTCTTTCTCTCAGCA 595 TCTCCACCCAACCAGCAGAAAACCGGTGAGTGGGGCTTPTAAGT TATTT TCAAGAAGAATGTAACAGATGTCAAACGGGAAAAGCACAÄGGCAAA---GCC

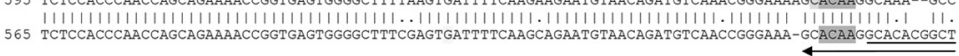
692 TGCTCTCTCTGTCTCTCTGTCTCCT-CTTCTCCTTI-TTTGCCT TATTCTATCCGATTTTT-TCC-CTAAGCTTCTACCTGGGATTTTCCTTTGGAAAAG

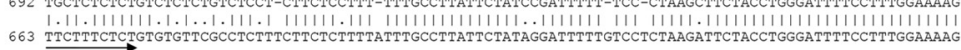
$789 \underset{\text { TGAGTTTGATGTTCCTTTGTITTCACTGTGATGTTAaTTTA GAaTAATACTACCTCTGATCCTAAAGCAAAGCAAAGCCTTACTGGCATGCCTGGGGAAA }}{\longrightarrow}$

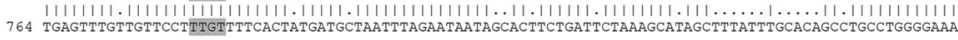
889 -TGTTTGCTGCTTGCCTTGAGGAGGTGGGGTCTCTTACCACTGCAGGTTG 864 ATGCTTGCTACTCATCTTGAGGGGTGG--CTCTTACTACTGCAGGTG

Figure 6. Regulation of the neuron-specific DCX-promoter by SoxC factors. $A$, SoxC proteins strongly enhance DCX promoter activity. The graph depicts DCX-luciferase activity induced by Sox2, Sox4, and Sox11, respectively, relative to control. $B$, The ability of Sox11 to transactivate the DCX promoter requires the region around and upstream of the TSS. Schematic diagram of the human genomic region upstream of the translation initiation codon. The TSS (angled arrows) and the relative positions of the putative CAAT and TATA box (vertical arrowheads) are shown above the deletion reporter constructs. Luciferase activities were normalized for CMV-Renilla--luciferase, and the response of each construct to Sox 11 was expressed as fold activation relative to the activity in the absence of the transcription factor. C, ChIP analysis showing binding of Sox 11 on the endogenous mouse DCX promoter. Schematic diagram of the mouse genomic region upstream of the translation initiation codon. The TSS (angled arrows) and the relative positions of the putative CAAT and TATA box (vertical arrowheads) are shown above the four amplicons analyzed in the ChIP assay. Black circles indicate the position of the putative Sox-binding sites in the Sox11-responsive region. ChIP assays were performed using adult NSCs. Chromatin extracts from cells that were transduced with a Sox 11 overexpressing virus or an EGFP control virus were precipitated using antibodies to Sox11, RNA Pol II or a nonspecific lgG control. The immunoprecipitated DNA was subjected (Figurelegend continues.) 
able antibodies did not precipitate endogenous Sox11 with high efficiency, we performed ChIP analysis from adult hippocampal NSCs overexpressing the Sox11 protein (see above). Cells overexpressing the EGFP protein were used as control. As shown in Figure $6 C$, the Sox11 antibody specifically precipitated DCXpromoter-containing chromatin fragments (amplicon B) only from Sox11-overexpressing cells. Interestingly, whereas the RNA Pol II antibody precipitated all four genomic fragments of the DCX promoter under basal conditions (i.e., in the EGFPoverexpressing control cells), in the presence of Sox11, the efficiency of precipitation of amplicons $\mathrm{B}$ and $\mathrm{C}$ was considerably higher (Fig. 6C). This observation indicates that Sox 11 is capable of enhancing the recruitment of Pol II to the proximal regions of the DCX promoter in which the TSS and the putative CAAT and TATA elements are located, thereby increasing the rate of synthesis of DCX mRNA. Finally, to begin to more precisely map the interaction of Sox 11 with the proximal DCX promoter region, we introduced two point mutations to the predicted SoxC binding site (-2037/-2031; CATTGCT to CAGGGCT, which corresponds to AGCAATG and AGCCCTG, respectively, on the minus strand) located between the putative CAAT and TATA elements (Fig. $6 D$ ) in the DCX reporter. This mutation resulted in a modest but significant reduction $(-31 \pm 5.7 \%, p<0.05)$ in DCX reporter activity in response to Sox 11 , suggesting that this predicted SoxC binding site may indeed contribute to the SoxCdependent regulation of the DCX promoter. Additional analyses, including electromobility shift assays and systematic mutation of all putative SoxC binding sites, however, are required to precisely map and identify the regulatory elements through which SoxC proteins control DCX expression.

Collectively, these data identify DCX as a direct target of SoxC transcription factors in adult NSCs and suggest a functional role of SoxC proteins in the control of a neuron-specific gene expression program.

\section{SoxC proteins are essential for reprogramming of astroglia into neurons}

Misexpression of lineage-specific factors represents a potent approach to induce cell-fate changes of somatic cells (Takahashi and Yamanaka, 2006; Vierbuchen et al., 2010). Expression of the pro-neural transcription factor Neurogenin 2 (Ngn2), for example, is sufficient to induce the conversion of astrocytes into glutamatergic neurons (Berninger et al., 2007; Heinrich et al., 2010, 2011). Reprogramming of somatic cells into neurons not only opens new avenues for cell-based regenerative medicine but also represents a stringent experimental system to study the contribution of transcription factors to neuronal fate determination and subtype specification (Heinrich et al., 2011). We exploited the Ngn2-induced neuronal conversion of astrocytes to further determine the role of Sox4/Sox 11 in neuronal differentiation. Astrocytes were isolated from cortices of P5-P7 mice. Cultured astrocytes were single transduced with retroviruses encoding for Ngn2 and dsRED (CAG-Ngn2-IRES-dsRED), for dsRED only (CAG-IRES-dsRED), the CAG-Sox4-IRES-GFP retrovirus,

\section{$\leftarrow$}

(Figure legend continued.) to quantitative $P C R$ using four different primer sets spanning the Sox11-responsive region of the $D C X$ promoter. The data were normalized to input chromatin and reported as relative fold enrichment over IgG immunoprecipitation. $D$, The Sox11-responsive region of the human DCX promoter is shown compared with the corresponding mouse sequence. The arrows indicate the position of the primers used for the ChIP. The core sequences of putative Sox-binding sites that are conserved between human and mouse are shown in gray. The putative CAAT and TATA boxes are in bold. and the CAG-Sox11-IRES-GFP retrovirus in different combinations. Consistent with previous reports (Berninger et al., 2007; Heinrich et al., 2010), we found that CAG-Ngn2-IRES-dsRED reprogrammed transduced astrocytes into DCX and $\beta$-tubulin III-positive neurons with an efficiency of 50 and 70\%, respectively (Fig. 7E). In contrast, Sox 4 and Sox 11 alone or in combination were insufficient to induce neuronal reprogramming (Fig. 7 and data not shown), which is in line with our recent observation that Sox 4 and Sox 11 are not sufficient to induce neuronal differentiation of proliferating precursor cells in a non-neurogenic region of the adult brain (data not shown). However, in combination with Ngn2, both SoxC transcription factors potently enhanced Ngn2-induced reprogramming of astrocytes into neurons with virtually all double-transduced cells displaying a complex neuronal morphology and expression of the neuronspecific markers $\beta$-tubulin III and DCX (Fig. 7). Next, we investigated whether SoxC transcription factors are necessary for neuronal reprogramming of astrocytes. To this end, we isolated astrocytes from cortices of P5-P7 Sox4/Sox11dcKO mice and mice heterozygous for the conditional Sox 4 and Sox11 alleles. Astrocyte cultures were transduced with the CAG-GFP-IRESCre in combination with CAG-Ngn2-IRES-dsRED or CAGIRES-dsRED. Cre-transduced Sox $4^{\text {loxP/+}}$; Sox $11^{\text {loxP/+ }}$ astrocytes were reprogrammed into $\beta$-tubulin III-positive neurons by Ngn2 at a rate comparable with wild-type cells $(68.2 \pm 1.4 \%)$ (Fig. $8 A, C)$. Strikingly, Ngn2-induced neuronal reprogramming was impaired in Cre-transduced Sox $4^{\text {loxP/loxP }}$; Sox $11^{\text {loxP/loxP }}$ astrocytes. In these cultures, cells not only showed strongly reduced expression of $\beta$-tubulin III $(18.7 \pm 1.6 \%)$ but also failed to develop a neuron-like morphology (Fig. $8 B, C$ ). Together, these findings demonstrate an essential role for Sox 4 and Sox 11 in neuronal reprogramming of astrocytes, thereby providing strong support for the notion of Sox 4 and Sox 11 as key contributors to the transcriptional network underlying neuronal differentiation.

\section{Discussion}

The present data identify the SoxC group transcription factors Sox 4 and Sox 11 as essential regulators of adult hippocampal neurogenesis and as crucial contributors to neuronal reprogramming of somatic cells. This conclusion is supported by the following observations. First, Sox 4 and Sox 11 protein expression is initiated in the adult hippocampal neurogenic lineage upon neuronal fate commitment. Second, Sox 4 and Sox 11 potently induce neurogenesis from adult hippocampal NSCs and promote neuronal reprogramming of astrocytes in combination with the pro-neural transcription factor Ngn2. Third, SoxC group proteins directly target and activate the promoter of the neuronal lineage-specific gene DCX. Fourth, loss of Sox 4 and Sox 11 abolishes expression of neuron-specific structural proteins in adult hippocampal neurogenesis and strongly impairs neuronal reprogramming of astrocytes.

The simultaneous expression of the transcription factors NeuroD1 and Prox 1 is specific for neuronal precursors and immature neurons of the dentate gyrus. NeuroD1 and Prox 1 transcription factors regulate dentate granule cell survival and maturation, respectively (Gao et al., 2009; Lavado et al., 2010; Karalay et al., 2011), and may cooperatively establish dentate granule subtypespecific traits. Sox4/Sox11 expression is initiated in parallel to NeuroD1 and Prox1 expression around the time of transition from a Sox $2^{+}$Type 2 a precursor cell toward a neuronally committed Type $2 b$ precursor cell stage (this study and Steiner et al., 2006; Lavado et al., 2010). What is the function of SoxC factors in adult hippocampal neurogenesis? In vitro, Sox 4 and Sox 11 were 

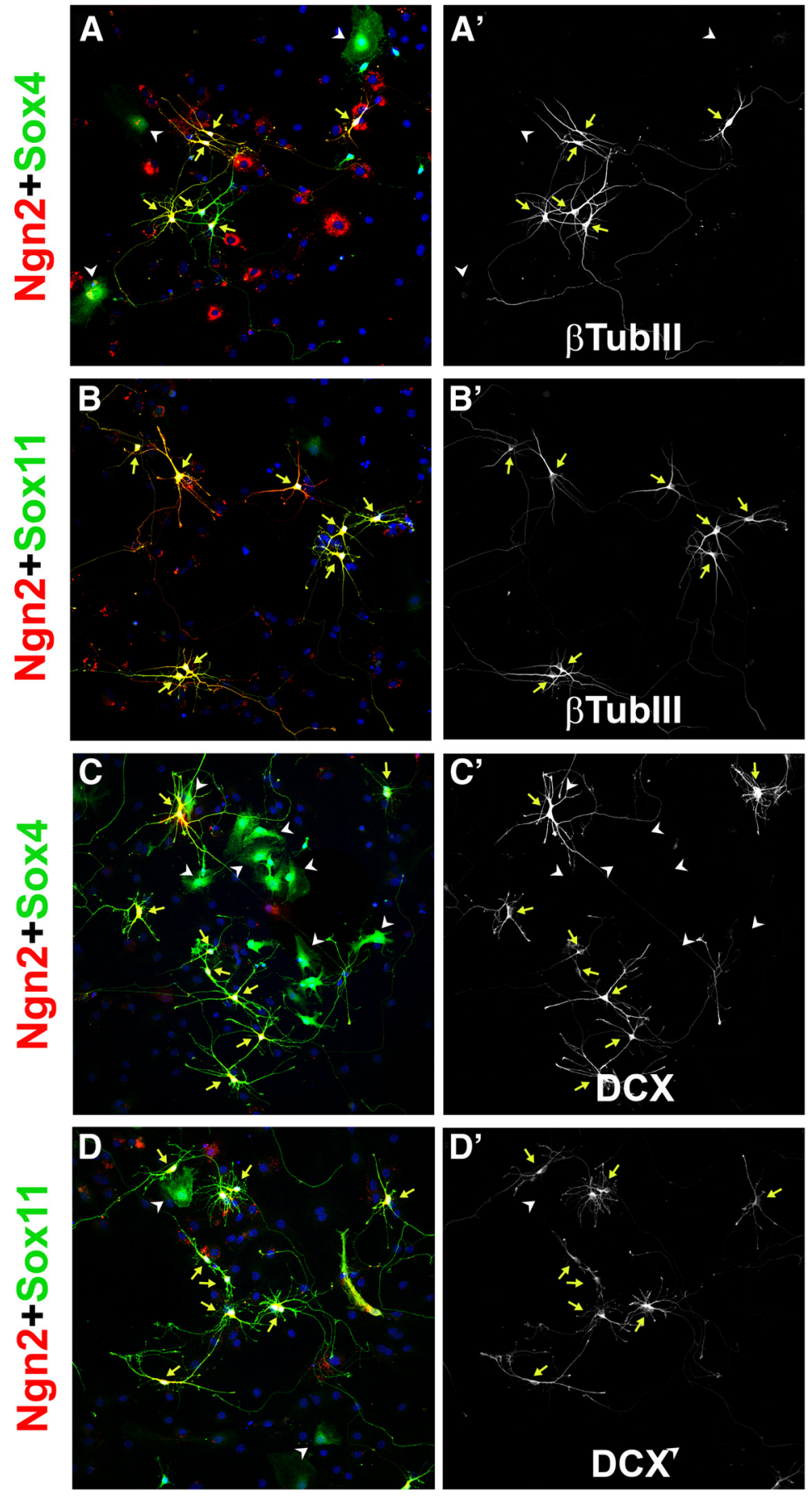

$\mathbf{E}$

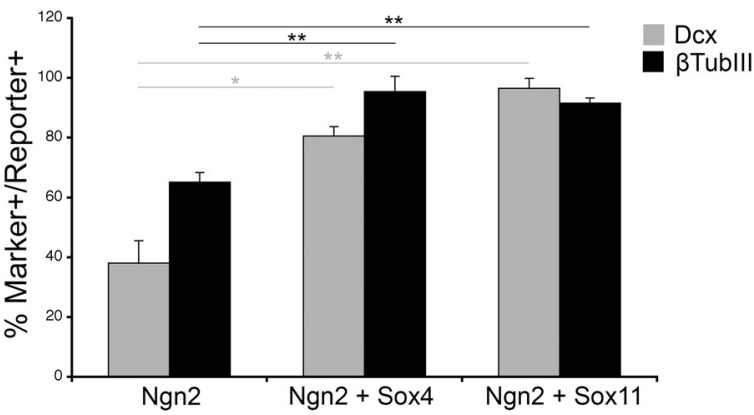

Figure 7. SoxC factors enhance Ngn2-induced astrocyte-to-neuron conversion. DsRed, GFP, and DAPI staining of P5-P7 astrocyte cultures $7 \mathrm{~d}$ after transduction with $\operatorname{Ngn} 2$ and $\operatorname{Sox} 4(\boldsymbol{A}, \boldsymbol{C})$ or Sox11 $(\boldsymbol{B}, \boldsymbol{D})$ and neuronal differentiation. Note that all the double-positive cells (yellow arrows) do express $\beta$-tubulin III (white, $\boldsymbol{A}^{\prime}, \boldsymbol{B}^{\prime}$ ) or DCX (white, $\boldsymbol{C}^{\prime}, \boldsymbol{D}^{\prime}$ ), whereas none of the cells transduced only with Sox $4-\mathrm{GFP}^{+}$or Sox $11-\mathrm{GFP}{ }^{+}$is $\beta$-tubulin III or DCX positive (white arrowheads). found to potently induce neuronal differentiation of proliferating adult hippocampal NSCs and to directly control the early neuron-specific gene DCX. In vivo, Sox4/Sox11 ablation decreased the generation of cells expressing neuronspecific proteins, without significant alterations in survival and proliferation. We therefore propose that Sox $4 /$ Sox 11 primarily regulate the initiation or maintenance of the neuronal differentiation program in the adult hippocampal neurogenic lineage, which is consistent with the observation that Sox11 targets neuron-specific promoters during the in vitro neuronal differentiation of mouse embryonic stem cells (Bergsland et al., 2011).

Notably, SoxC transcription factors seem to be expressed by all immature neurons in both adult neurogenic regions (Haslinger et al., 2009) and throughout the developing mammalian nervous system regardless of their neuronal subtype identity (Hargrave et al., 1997; Cheung et al., 2000; Sock et al., 2004). It is also of note that SoxC targets comprise a number of structural proteins, such as DCX (this study), $\beta$-tubulin III (Bergsland et al., 2006,2011 ), and the F-actin binding protein drebrin (Song et al., 2008; Wang et al., 2010), that are expressed by immature neurons of many, if not all, subtype lineages during adult and embryonic neurogenesis. It therefore seems likely that SoxC proteins-in analogy to their function in chicken spinal cord development (Bergsland et al., 2006, 2011) — control the expression of pan-neuronal traits rather than the expression dentate granule neuron-specific genetic programs in the adult hippocampal neurogenic lineage. It is, however, important to mention that expression of the SoxC target DCX can be modulated by NeuroD1 (Seo et al., 2007; Zheng et al., 2010) and Proxl (Lavado et al., 2010) and that SoxC proteins can modulate Wnt-signaling activity (Sinner et al., 2007; Lee et al., 2011), which operates upstream of NeuroD1 and Prox1 (Kuwabara et al., 2009; Karalay et al., 2011). Hence, crosstalk between SoxCdependent pathways with the Wnt/NeuroD1/Prox1 pathway may be essential to orchestrate the development of new dentate granule neurons.

The function of the third member of the SoxC protein family, i.e., Sox12, was

E, Quantification of $\beta$-tubulin $\mathrm{II}^{+}$and $\mathrm{DCX}{ }^{+}$neurons among transduced astrocytes. 
not examined in the present study. Sox 12 is a weak transactivator compared with Sox 4 and Sox 11 and seems to be dispensable for mouse embryonic development (Hoser et al., 2008). In situ hybridization data available from the Allen Brain Atlas project (http://www.brain-map.org/) indicates that Sox12 is coexpressed with Sox 4 and Sox11 in the adult hippocampus. Lack of suitable antibodies precluded detailed analysis of Sox 12 protein expression in the adult hippocampal neurogenic lineage. The finding that Sox4/Sox11 deletion was sufficient to abolish neuronal differentiation, however, demonstrates that Sox12 does not compensate for loss of Sox4/Sox11 function in adult hippocampal neurogenesis, which is consistent with the observation that Sox12 is unable to compensate the effects of Sox 4 and Sox 11 deletion during embryonic development (Hoser et al., 2008; Bhattaram et al., 2010).

How SoxC expression in the adult neurogenic lineage is controlled remains to be determined. In the developing chicken spinal cord, SoxC operate downstream of neurogenins (Bergsland et al., 2006). Indeed, our preliminary studies revealed induction of SoxC mRNA expression on Ngn2-mediated reprogramming of astrocytes (G.M. and B.B., unpublished observations). Moreover, Ngn2 expression is found in Type 2 a precursor cells of the early postnatal and adult dentate gyrus (Ozen et al., 2007; Roybon et al., 2009b) and thus precedes the induction of SoxC protein expression. Hence, Ngn2 represents a promising candidate to control SoxC expression in the adult hippocampal neurogenic lineage. It is, however, important to note that SoxC transcription factors are expressed also in newborn neurons in adult subventricular zone/olfactory bulb neurogenesis (Haslinger et al., 2009). Here, only a minor fraction of these neurons are generated from Ngn2-expressing precursors (Brill et al., 2009; Roybon et al., 2009a). Thus, transcriptional activation of SoxC genes in neurogenesis may differ between the adult neurogenic regions and may be controlled by additional factors such as Mash1, which is expressed by early precursors in both neurogenic lineages (Kim et al., 2011) and influences SoxC gene expression in the developing brain (Castro et al., 2011). Finally, the observation that SoxC mRNA expression is already observed in SoxC-protein-negative adult NSCs (Beckervordersandforth et al., 2010) indicates that posttranscriptional mechanisms play a significant role in the expression of SoxC proteins and SoxCdependent neuronal differentiation.

It has been shown that Sox proteins require cooperation with other transcription factors for efficient transactivation of target genes (Kondoh and Kamachi, 2010). It is therefore likely that SoxC factors act in concert with currently unknown factors to control neuron-specific gene expression programs. In silico anal-

\section{Ngn2+Cre}
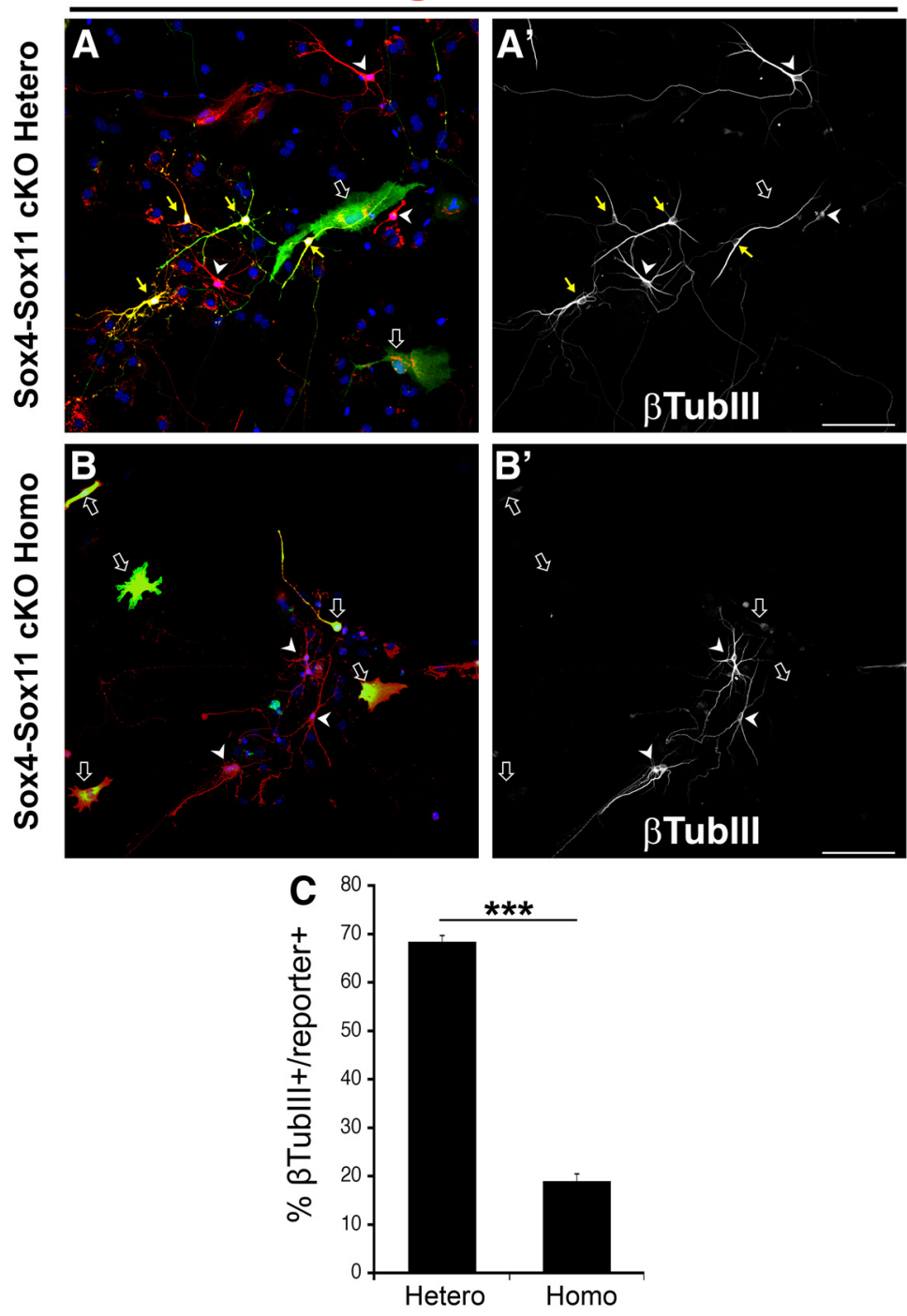

Figure 8. SoxC factors are essential for astrocyte-to-neuron conversion. $\boldsymbol{A}, \boldsymbol{A}^{\prime}$, Sox $4^{\text {flox } /+}$; Sox $17^{f l o x /+}$ postnatal astrocytes cotransduced with retroviruses encoding Ngn2 (in red) and (re recombinase (in green) ( $\boldsymbol{A}$, yellow arrows) generated $\beta$-tubulin $I^{+}$neurons $\left(\boldsymbol{A}^{\prime}\right)$. On the contrary, Sox $4^{\text {flox/flox }}$; Sox $17^{\text {flox/flox }}$ postnatal astrocytes, transduced with the same viruses, did not induce presence of Ngn2-Cre double-transduced cells that did not give rise to neurons (white open arrow). C, Quantification of $\beta$-tubulin III-positive neurons in Sox4 -Sox11 heterozygous and homozygous postnatal astrocytes on Ngn2 and Cre transduction.

ysis of highly conserved noncoding DNA regions revealed that regulatory regions of developmentally expressed CNS genes are enriched for Sox, POU, and homeodomain transcription factor binding sites (Bailey et al., 2006). It has also been reported that Brn proteins, which belong to the POU family of transcription factors and which are predominantly expressed in the neuronal lineage, enhance the activation of SoxC target genes (Kuhlbrodt et al., 1998; Wiebe et al., 2003; Tanaka et al., 2004). Intriguingly, the DCX regulatory region contains several conserved putative binding sites for Brn transcription factors (Karl et al., 2005) that are located in close proximity to the Sox 11 binding sites identified in this study. It will be interesting to determine the interaction of Brn proteins with SoxC factors and its functional contribution in the control of neuronal differentiation in adult neurogenesis. In addition to the identification of SoxC interacting partners, it will also be interesting to decipher the downstream targets of the SoxC transcription factor network in differentiating adult NSCs. 
These analyses will result in a more complete understanding of the regulatory network controlling adult NSC differentiation and neuronal cell identity and may also identify new candidate targets for the efficient neuronal reprogramming of somatic cells in regenerative medicine.

\section{References}

Bailey PJ, Klos JM, Andersson E, Karlén M, Källström M, Ponjavic J, Muhr J, Lenhard B, Sandelin A, Ericson J (2006) A global genomic transcriptional code associated with CNS-expressed genes. Exp Cell Res 312: 3108-3119.

Beckervordersandforth R, Tripathi P, Ninkovic J, Bayam E, Lepier A, Stempfhuber B, Kirchhoff F, Hirrlinger J, Haslinger A, Lie DC, Beckers J, Yoder B, Irmler M, Götz M (2010) In vivo fate mapping and expression analysis reveals molecular hallmarks of prospectively isolated adult neural stem cells. Cell Stem Cell 7:744-758.

Bergsland M, Werme M, Malewicz M, Perlmann T, Muhr J (2006) The establishment of neuronal properties is controlled by Sox4 and Sox11. Genes Dev 20:3475-3486.

Bergsland M, Ramsköld D, Zaouter C, Klum S, Sandberg R, Muhr J (2011) Sequentially acting Sox transcription factors in neural lineage development. Genes Dev 25:2453-2464.

Berninger B, Costa MR, Koch U, Schroeder T, Sutor B, Grothe B, Götz M (2007) Functional properties of neurons derived from in vitro reprogrammed postnatal astroglia. J Neurosci 27:8654-8664.

Bhattaram P, Penzo-Méndez A, Sock E, Colmenares C, Kaneko KJ, Vassilev A, Depamphilis ML, Wegner M, Lefebvre V (2010) Organogenesis relies on SoxC transcription factors for the survival of neural and mesenchymal progenitors. Nat Commun 1:9.

Brandt MD, Jessberger S, Steiner B, Kronenberg G, Reuter K, Bick-Sander A, von der Behrens W, Kempermann G (2003) Transient calretinin expression defines early postmitotic step of neuronal differentiation in adult hippocampal neurogenesis of mice. Mol Cell Neurosci 24:603-613.

Brill MS, Ninkovic J, Winpenny E, Hodge RD, Ozen I, Yang R, Lepier A, Gascón S, Erdelyi F, Szabo G, Parras C, Guillemot F, Frotscher M, Berninger B, Hevner RF, Raineteau O, Götz M (2009) Adult generation of glutamatergic olfactory bulb interneurons. Nat Neurosci 12:1524-1533.

Castro DS, Martynoga B, Parras C, Ramesh V, Pacary E, Johnston C, Drechsel D, Lebel-Potter M, Garcia LG, Hunt C, Dolle D, Bithell A, Ettwiller L, Buckley N, Guillemot F (2011) A novel function of the proneural factor Ascll in progenitor proliferation identified by genome-wide characterization of its targets. Genes Dev 25:930-945.

Cheung M, Abu-Elmagd M, Clevers H, Scotting PJ (2000) Roles of Sox4 in central nervous system development. Brain Res Mol Brain Res 79:180-191.

Couillard-Despres S, Winner B, Karl C, Lindemann G, Schmid P, Aigner R, Laemke J, Bogdahn U, Winkler J, Bischofberger J, Aigner L (2006) Targeted transgene expression in neuronal precursors: watching young neurons in the old brain. Eur J Neurosci 24:1535-1545.

Couillard-Despres S, Finkl R, Winner B, Ploetz S, Wiedermann D, Aigner R, Bogdahn U, Winkler J, Hoehn M, Aigner L (2008) In vivo optical imaging of neurogenesis: watching new neurons in the intact brain. Mol Imaging 7:28-34.

Ehm O, Göritz C, Covic M, Schäffner I, Schwarz TJ, Karaca E, Kempkes B, Kremmer E, Pfrieger FW, Espinosa L, Bigas A, Giachino C, Taylor V, Frisén J, Lie DC (2010) RBPJ $\kappa$-dependent signaling is essential for longterm maintenance of neural stem cells in the adult hippocampus. J Neurosci 30:13794-13807.

Ellis P, Fagan BM, Magness ST, Hutton S, Taranova O, Hayashi S, McMahon A, Rao M, Pevny L (2004) SOX2, a persistent marker for multipotential neural stem cells derived from embryonic stem cells, the embryo or the adult. Dev Neurosci 26:148-165.

Favaro R, Valotta M, Ferri AL, Latorre E, Mariani J, Giachino C, Lancini C, Tosetti V, Ottolenghi S, Taylor V, Nicolis SK (2009) Hippocampal development and neural stem cell maintenance require Sox2-dependent regulation of Shh. Nat Neurosci 12:1248-1256.

Gao Z, Ure K, Ables JL, Lagace DC, Nave KA, Goebbels S, Eisch AJ, Hsieh J (2009) Neurod1 is essential for the survival and maturation of adultborn neurons. Nat Neurosci 12:1090-1092.

Hargrave M, Wright E, Kun J, Emery J, Cooper L, Koopman P (1997) Ex- pression of the Sox11 gene in mouse embryos suggests roles in neuronal maturation and epithelio-mesenchymal induction. Dev Dyn 210:79-86.

Harley VR, Lovell-Badge R, Goodfellow PN (1994) Definition of a consensus DNA binding site for SRY. Nucleic Acids Res 22:1500-1501.

Haslinger A, Schwarz TJ, Covic M, Chichung Lie D (2009) Expression of Sox11 in adult neurogenic niches suggests a stage-specific role in adult neurogenesis. Eur J Neurosci 29:2103-2114.

Heinrich C, Blum R, Gascón S, Masserdotti G, Tripathi P, Sánchez R, Tiedt S, Schroeder T, Götz M, Berninger B (2010) Directing astroglia from the cerebral cortex into subtype specific functional neurons. PLoS Biol 8:e1000373.

Heinrich C, Gascón S, Masserdotti G, Lepier A, Sanchez R, Simon-Ebert T, Schroeder T, Götz M, Berninger B (2011) Generation of subtypespecific neurons from postnatal astroglia of the mouse cerebral cortex. Nat Protoc 6:214-228

Hodge RD, Kowalczyk TD, Wolf SA, Encinas JM, Rippey C, Enikolopov G, Kempermann G, Hevner RF (2008) Intermediate progenitors in adult hippocampal neurogenesis: Tbr2 expression and coordinate regulation of neuronal output. J Neurosci 28:3707-3717.

Hoser M, Potzner MR, Koch JM, Bösl MR, Wegner M, Sock E (2008) Sox12 deletion in the mouse reveals nonreciprocal redundancy with the related Sox4 and Sox11 transcription factors. Mol Cell Biol 28:4675-4687.

Jagasia R, Steib K, Englberger E, Herold S, Faus-Kessler T, Saxe M, Gage FH, Song H, Lie DC (2009) GABA-cAMP response element-binding protein signaling regulates maturation and survival of newly generated neurons in the adult hippocampus. J Neurosci 29:7966-7977.

Karalay O, Doberauer K, Vadodaria KC, Knobloch M, Berti L, Miquelajauregui A, Schwark M, Jagasia R, Taketo MM, Tarabykin V, Lie DC, Jessberger S (2011) Prospero-related homeobox 1 gene (Prox1) is regulated by canonical Wnt signaling and has a stage-specific role in adult hippocampal neurogenesis. Proc Natl Acad Sci U S A 108:5807-5812.

Karl C, Couillard-Despres S, Prang P, Munding M, Kilb W, Brigadski T, Plötz S, Mages W, Luhmann H, Winkler J, Bogdahn U, Aigner L (2005) Neuronal precursor-specific activity of a human doublecortin regulatory sequence. J Neurochem 92:264-282.

Kim EJ, Ables JL, Dickel LK, Eisch AJ, Johnson JE (2011) Ascl1 (Mash1) defines cells with long-term neurogenic potential in subgranular and subventricular zones in adult mouse brain. PLoS One 6:e18472.

Kondoh H, Kamachi Y (2010) SOX-partner code for cell specification: Regulatory target selection and underlying molecular mechanisms. Int J Biochem Cell Biol 42:391-399.

Kuhlbrodt K, Herbarth B, Sock E, Enderich J, Hermans-Borgmeyer I, Wegner M (1998) Cooperative function of POU proteins and SOX proteins in glial cells. J Biol Chem 273:16050-16057.

Kuwabara T, Hsieh J, Muotri A, Yeo G, Warashina M, Lie DC, Moore L, Nakashima K, Asashima M, Gage FH (2009) Wnt-mediated activation of NeuroD1 and retro-elements during adult neurogenesis. Nat Neurosci 12:1097-1105.

Lavado A, Lagutin OV, Chow LM, Baker SJ, Oliver G (2010) Prox1 is required for granule cell maturation and intermediate progenitor maintenance during brain neurogenesis. PLoS Biol 8:pii:e1000460.

Lee AK, Ahn SG, Yoon JH, Kim SA (2011) Sox4 stimulates ss-catenin activity through induction of CK2. Oncol Rep 25:559-565.

Lie DC, Colamarino SA, Song HJ, Désir é L, Mira H, Consiglio A, Lein ES, Jessberger S, Lansford H, Dearie AR, Gage FH (2005) Wnt signalling regulates adult hippocampal neurogenesis. Nature 437:1370-1375.

Ozen I, Galichet C, Watts C, Parras C, Guillemot F, Raineteau O (2007) Proliferating neuronal progenitors in the postnatal hippocampus transiently express the proneural gene Ngn2. Eur J Neurosci 25:2591-2603.

Penzo-Méndez A, Dy P, Pallavi B, Lefebvre V (2007) Generation of mice harboring a Sox4 conditional null allele. Genesis 45:776-780.

Pevny L, Placzek M (2005) SOX genes and neural progenitor identity. Curr Opin Neurobiol 15:7-13.

Potzner MR, Griffel C, Lütjen-Drecoll E, Bösl MR, Wegner M, Sock E (2007) Prolonged Sox4 expression in oligodendrocytes interferes with normal myelination in the central nervous system. Mol Cell Biol 27:5316-5326.

Potzner MR, Tsarovina K, Binder E, Penzo-Méndez A, Lefebvre V, Rohrer H, Wegner M, Sock E (2010) Sequential requirement of Sox4 and Sox11 during development of the sympathetic nervous system. Development 137:775-784.

Ray J, Gage FH (2006) Differential properties of adult rat and mouse brainderived neural stem/progenitor cells. Mol Cell Neurosci 31:560-573. 
Rottach A, Kremmer E, Nowak D, Leonhardt H, Cardoso MC (2008) Generation and characterization of a rat monoclonal antibody specific for multiple red fluorescent proteins. Hybridoma (Larchmt) 27:337-343.

Roybon L, Deierborg T, Brundin P, Li JY (2009a) Involvement of Ngn2, Tbr and NeuroD proteins during postnatal olfactory bulb neurogenesis. Eur J Neurosci 29:232-243.

Roybon L, Hjalt T, Stott S, Guillemot F, Li JY, Brundin P (2009b) Neurogenin2 directs granule neuroblast production and amplification while NeuroD1 specifies neuronal fate during hippocampal neurogenesis. PLoS One 4:e4779.

Seo S, Lim JW, Yellajoshyula D, Chang LW, Kroll KL (2007) Neurogenin and NeuroD direct transcriptional targets and their regulatory enhancers. EMBO J 26:5093-5108.

Sinner D, Kordich JJ, Spence JR, Opoka R, Rankin S, Lin SC, Jonatan D, Zorn AM, Wells JM (2007) Sox17 and Sox4 differentially regulate betacatenin/TCF activity and proliferation of colon carcinoma cells. Mol Cell Biol 27:7802-7815.

Sock E, Rettig SD, Enderich J, Bösl MR, Tamm ER, Wegner M (2004) Gene targeting reveals a widespread role for the high-mobility-group transcription factor Sox11 in tissue remodeling. Mol Cell Biol 24:6635-6644.

Song M, Kojima N, Hanamura K, Sekino Y, Inoue HK, Mikuni M, Shirao T (2008) Expression of drebrin $\mathrm{E}$ in migrating neuroblasts in adult rat brain: coincidence between drebrin E disappearance from cell body and cessation of migration. Neuroscience 152:670-682.

Steiner B, Klempin F, Wang L, Kott M, Kettenmann H, Kempermann G (2006) Type-2 cells as link between glial and neuronal lineage in adult hippocampal neurogenesis. Glia 54:805-814.

Steiner B, Zurborg S, Hörster H, Fabel K, Kempermann G (2008) Differential $24 \mathrm{~h}$ responsiveness of Proxl-expressing precursor cells in adult hippocampal neurogenesis to physical activity, environmental enrichment, and kainic acid-induced seizures. Neuroscience 154:521-529.

Suh H, Consiglio A, Ray J, Sawai T, D’Amour KA, Gage FH (2007) In vivo fate analysis reveals the multipotent and self-renewal capacities of Sox $2^{+}$ neural stem cells in the adult hippocampus. Cell Stem Cell 1:515-528.

Takahashi K, Yamanaka S (2006) Induction of pluripotent stem cells from mouse embryonic and adult fibroblast cultures by defined factors. Cell 126:663-676

Tanaka S, Kamachi Y, Tanouchi A, Hamada H, Jing N, Kondoh H (2004) Interplay of SOX and POU factors in regulation of the Nestin gene in neural primordial cells. Mol Cell Biol 24:8834-8846.

Tashiro A, Zhao C, Gage FH (2006) Retrovirus-mediated single-cell gene knockout technique in adult newborn neurons in vivo. Nat Protoc 1:3049-3055.

Thein DC, Thalhammer JM, Hartwig AC, Crenshaw EB 3rd, Lefebvre V, Wegner M, Sock E (2010) The closely related transcription factors Sox4 and Sox11 function as survival factors during spinal cord development. J Neurochem 115:131-141.

Vierbuchen T, Ostermeier A, Pang ZP, Kokubu Y, Südhof TC, Wernig M (2010) Direct conversion of fibroblasts to functional neurons by defined factors. Nature 463:1035-1041.

Wang X, Björklund S, Wasik AM, Grandien A, Andersson P, Kimby E, Dahlman-Wright K, Zhao C, Christensson B, Sander B (2010) Gene expression profiling and chromatin immunoprecipitation identify DBN1, SETMAR and HIG2 as direct targets of SOX11 in mantle cell lymphoma. PLoS One 5:e14085.

Wiebe MS, Nowling TK, Rizzino A (2003) Identification of novel domains within Sox-2 and Sox-11 involved in autoinhibition of DNA binding and partnership specificity. J Biol Chem 278:17901-17911.

Zhang J, Giesert F, Kloos K, Vogt Weisenhorn DM, Aigner L, Wurst W, CouillardDespres S (2010) A powerful transgenic tool for fate mapping and functional analysis of newly generated neurons. BMC Neurosci 11:158.

Zhao C, Teng EM, Summers RG Jr, Ming GL, Gage FH (2006) Distinct morphological stages of dentate granule neuron maturation in the adult mouse hippocampus. J Neurosci 26:3-11.

Zhao C, Deng W, Gage FH (2008) Mechanisms and functional implications of adult neurogenesis. Cell 132:645-660.

Zheng H, Zeng Y, Chu J, Kam AY, Loh HH, Law PY (2010) Modulations of NeuroD activity contribute to the differential effects of morphine and fentanyl on dendritic spine stability. J Neurosci 30:8102-8110. 